Research Article

\title{
FT-IR, Laser-Raman, UV-Vis, and NMR Spectroscopic Studies of Antidiabetic Molecule Nateglinide
}

\author{
Tuba Özdemir Öge (i) \\ Vocational School of Health Services, Bartın University, 74100 Bartın, Turkey \\ Correspondence should be addressed to Tuba Özdemir Öge; tozdemir@bartin.edu.tr
}

Received 5 June 2018; Accepted 26 August 2018; Published 24 September 2018

Academic Editor: Rizwan Hasan Khan

Copyright ( 2018 Tuba Özdemir Öge. This is an open access article distributed under the Creative Commons Attribution License, which permits unrestricted use, distribution, and reproduction in any medium, provided the original work is properly cited.

The quantum chemical calculations and spectroscopic and theoretical characterizations of nateglinide molecule, a derivative of meglitinide and an oral antidiabetic drug, were performed using FT-IR, Laser-Raman, and NMR chemical shift and UV-Vis analysis methods. The other parameters including geometric structures, optimized geometry, vibrational frequencies, dipole moments, infrared and Raman intensities, and HOMO and LUMO energies of nateglinide molecules were studied using the density functional theory. In addition, the ${ }^{13} \mathrm{C}$ and ${ }^{1} \mathrm{H}$ NMRs were calculated using Gaussian 09 program with the DFT/B3LYP method at the 6-31G + (d,p) basis set. TD-DFT calculations were performed to examine the electronic transitions including orbital energies, absorption wavelengths, oscillator strengths, and excitation energies in methanol. The research was performed to provide detailed spectroscopic information of antidiabetic nateglinide molecule's monomer conformations.

\section{Introduction}

Meglitinides (glitinides) are insulin secretagogues, and they stimulate insulin release from pancreas [1-3]. Nateglinide, a derivative of meglitinide, is an oral antidiabetic drug used for the treatment of type II diabetes mellitus. Nateglinide lowers the blood glucose levels by stimulating insulin secretion from the pancreas. The structure name of nateglinide is (-)-N-[(trans-4-isopropylcyclohexane) carbonyl $]-\mathrm{D}$ phenylalanine [4-7]. The chemical formula of nateglinide used in the present study is $\mathrm{C}_{19} \mathrm{H}_{27} \mathrm{NO}_{3}$. The CAS and MDL numbers are 105816-04-4 and MFCD00875706, respectively. The molecular weight of nateglinide is $317.42 \mathrm{~g} / \mathrm{mol}$. The synonyms of nateglinide are given as Fastic, $N$ - $[$ trans-4isopropylcyclohexyl)carbonyl]-D-phenylalanine, Starlix, and Starsis in [8].

Jain et al. carried out a work on spectrophotometric determination of nateglinide in bulk and tablet dosage forms [9]. Babu et al. investigated nateglinide with visible spectrophotometry and spectrophotometric methods $[10,11]$. Bruni et al. developed a method for the quantification of the polymorphic purity of nateglinide in mixtures formed by polymorphs $\mathrm{H}$ and B [12]. Guardado-Mendoza et al. explained that nateglinide and repaglinide are effective in reducing postprandial glucose excursion and $\mathrm{HbAlc}$ levels from $0.8 \%$ to $1 \%$ in T2DM [13]. Rani et al. described two spectrophotometric methods for the determination of nateglinide [14]. Goyal et al. determined the crystal structure and crystallographic parameters of experimentally crystallized polymorphs of nateglinide. Crystallographic parameters of nateglinide polymorphs were given as Form $\mathrm{H}$, Form B, Form MS, and Form S (space groups are P-1, C2, P-4, and $\mathrm{P}-42 \mathrm{C}$ ), and the forms were found to exist in triclinic, monoclinic, tetragonal, and tetragonal, correspondingly [15]. Remko [16] used the methods of theoretical chemistry to elucidate the molecular properties of the hypoglycemic sulfonylureas and glinides (acetohexamide, tolazamide, tolbutamide, chlorpropamide, gliclazide, glimepiride, glipizide, glibenclamide, nateglinide, and repaglinide) which are known as antidiabetic molecules. The geometry and energy of these drugs were computed using the Becke3LYP/6-31G $+(d, p)$ method. Karakaya et al. [17] investigated the vibrational and structural properties of tolazamide molecule. Ozdemir and Gokce studied the glimepiride molecule as a sulfonylurea compound using FT-IR, Raman and NMR spectroscopy, and DFT theory [18]. 
Few studies have been encountered on investigation of nateglinide molecule, which is effectively used against diabetes mellitus. In this regard, the present research was carried out to perform a detailed theoretical and experimental investigation of nateglinide, which is the active ingredient of an antidiabetic drug commonly prescribed as Starlix, using spectroscopic analyses such as FT-IR, LaserRaman, UV-Vis, and NMR. In the present study, density functional theory study was theoretically performed to obtain the vibrational wavenumbers, FT-IR, Laser-Raman, and NMR chemical shifts and UV-Vis of nateglinide molecule. The recorded experimental data were supported with the computed parameters using theoretical methods at DFT/B3LYP/6-31G + (d, p) level. The obtained theoretical and experimental results were used to give detailed information of the molecular electronic structure of nateglinide.

\section{Experimental and Computational Procedures}

Nateglinide was purchased from Sigma-Aldrich Corporation in powder form. The melting point of nateglinide is in the range of $137^{\circ} \mathrm{C}-141^{\circ} \mathrm{C}$. The chemical name of nateglinide (NTG) is $\mathrm{N}$-(trans-4-isopropylcyclohexyl carbonyl)-Dphenylalanine. This molecule is almost insoluble in water, and highly soluble in methylene and methanol chloride. It shows polymorphism [19]. The optimized monomer structures of antidiabetic molecule are given in Figure 1. The FT-IR spectrum of nateglinide molecule was recorded within $400-4000 \mathrm{~cm}^{-1}$ region at room temperature, using the potassium bromide $(\mathrm{KBr})$ pellet, on a Fourier-transform infrared spectrometer in the solid phase of the sample as shown in Figure 2. The Laser-Raman spectrum was recorded at room temperature in $100-4000 \mathrm{~cm}^{-1}$ region as shown in Figure 3. The ${ }^{1} \mathrm{H}$ and ${ }^{13} \mathrm{C}$ NMR chemical shift spectra of the compound solved in dimethyl sulfoxide (DMSO-d6) were recorded with TMS as the internal standard using the Premium Compact NMR device at $600 \mathrm{MHz}$ frequency and 14.1 Tesla field power. The chemical shifts were reported at ppm level as given in Figures 4 and 5. The ultraviolet visible spectrum of nateglinide dissolved in methanol was recorded using a UV-Vis spectrophotometer in 200-400 nm range at room temperature as given in Figure 6.

B3LYP (Becke, three-parameter, Lee-Yang-Parr) level with $6-31 G+(d, p)$ basis set was used to compute the electronic structure properties of nateglinide molecule $[20,21]$. Vibrational wavenumbers, geometric parameters, and molecular properties were calculated using Gaussian 09W software and GaussView5 molecular visualization program on a computer system [22-24]. Veda 4 program was used to compute the potential energy distribution of vibrational wavenumbers as given in Table 1 [25]. The major contributions for the computed electronic wavelengths were obtained by GaussSum 3.0 program as listed in Table 2 [26].

\section{Results and Discussion}

3.1. Geometric Structure. The experimental data explain the crystallographic structure of nateglinide [27], and these findings were compared with the calculated results as given in Table 3. The other geometric parameters such as bond lengths, bond angles, and torsion angles with the corresponding literature information are given in Table 3. Zeropoint, relative energy values, and dipole moments are given in Table 4. Tessler and Goldberg investigated bis(nateglinide) hydronium chloride, in addition to its self-assembly into extended polymeric arrays with $\mathrm{O}-\mathrm{H} \cdots \mathrm{O}, \mathrm{N}-\mathrm{H} \cdots \mathrm{Cl}$, and $\mathrm{O}-\mathrm{H} \cdots \mathrm{Cl}$ hydrogen bonds. The title compound contains four dissimilar moieties which are conformationally different in the asymmetric unit [27].

The C-C bond lengths of the title molecule were calculated at the interval of $1.395-1.558 \AA$, while they were recorded between 1.352 and $1.543 \AA$ in the literature [27]. The C-N bond lengths of the title molecule were calculated at $1.447 \AA$ and $1.372 \AA$, while they were recorded at $1.460 \AA$ and 1.332 in the literature [27]. In this research, the $\mathrm{C}-\mathrm{H}$ bond lengths of the title molecule were calculated at the interval of 1.086-1.103 A. The calculated C11-C13, C13-C15, C9-C11, C7-C9, C7-C17, C15-C17, C17-C18, C18-C21, C21-C23, C24-C25, C25-C38, C35-C38, C33-C35, C33-C30, C27C30, C25-C27, C33-C41, C41-C47, and C41-C43 bond lengths for nateglinide are $1.398 \AA$, $1.395 \AA, 1.396 \AA, 1.398 \AA$, $1.401 \AA, 1.403 \AA, 1.514 \AA, 1.558 \AA, 1.525 \AA, 1.530 \AA, 1.546 \AA$, $1.537 \AA, 1.542 \AA, 1.542 \AA, 1.539 \AA, 1.539 \AA, 1.554 \AA, 1.539 \AA$, and $1.539 \AA$, respectively. The calculated bond angles of C24C25-C27, C24-C25-C38, C25-C27-C30, C25-C38-C35, C38C35-C33, C35-C33-C30, and C33-C30-C27 are $117.171^{\circ}$, $109.582^{\circ}, 110.933^{\circ}, 111.408^{\circ}, 112.186^{\circ}, 109.752^{\circ}$, and $112.163^{\circ}$, respectively, in this study. The calculated dihedral angle of C9-C7-C17-C18 is $-179.489^{\circ}$.

Jain et al. studied the monomers, dimers, and tetramers of nateglinide to understand the conformational properties. Nateglinide molecule contains two strong hydrogen bond donors as $\mathrm{N}-\mathrm{H} / \mathrm{O}-\mathrm{H}$ and two strong acceptors as $2 \times \mathrm{C}=\mathrm{O}$ [28]. As in the present research, two conformations come into prominence, namely, N-A and N-C. These two differ from others by the relative position of hydrogen in the carboxylic group and by torsional angle across C2-C3$\mathrm{N} 4-\mathrm{C} 5\left(-169.17^{\circ}\right.$ in $\mathrm{N}-\mathrm{A}$ and $-118.57^{\circ}$ in N-C). Likewise, the torsional angles of Monomer 1 and Monomer 2 were calculated $-169.15007^{\circ}$ and $-120.87747^{\circ}$, respectively, in the present research.

3.2. Vibrational Frequency Analyses. In the following discussion, nateglinide is experimentally examined using FTIR, Laser-Raman, UV-Vis spectroscopy, and NMR. The observed and calculated vibrational frequencies, observed and calculated FT-IR intensities, Raman scattering activities, and vibrational assignments of the title molecule are given in Table 1. Nateglinide consists of 50 atoms, and accordingly, it has 144 modes of vibrations according to the relation $3 \mathrm{~N}-6$ (for $\mathrm{N}=50$ ). In the present research, C-H, C-O, O-H, N-H, and $\mathrm{C}-\mathrm{C}$ vibrations were examined. As shown in Figures 2 and 3 and Table 1 , the experimental and calculated vibrational wavenumbers are in good agreement.

The computations of harmonic wavenumbers, IR intensities, and Raman activities were performed with the 


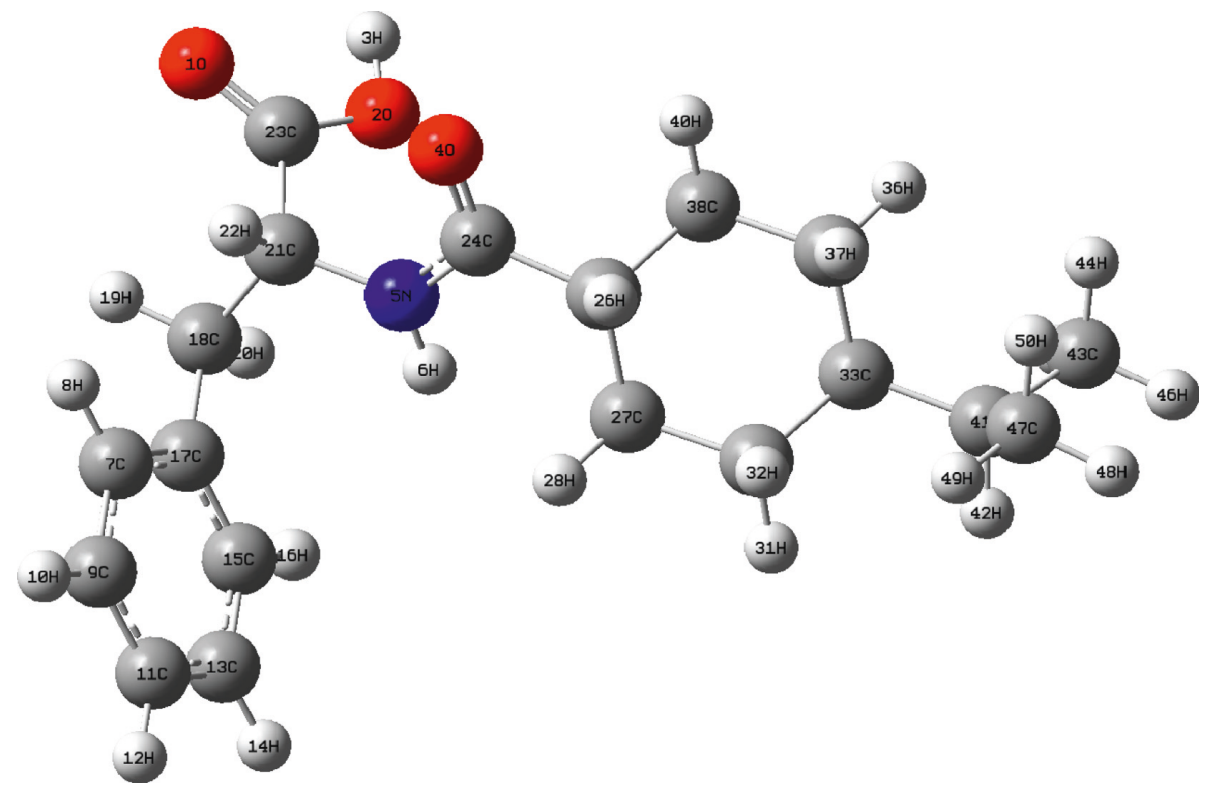

(a)

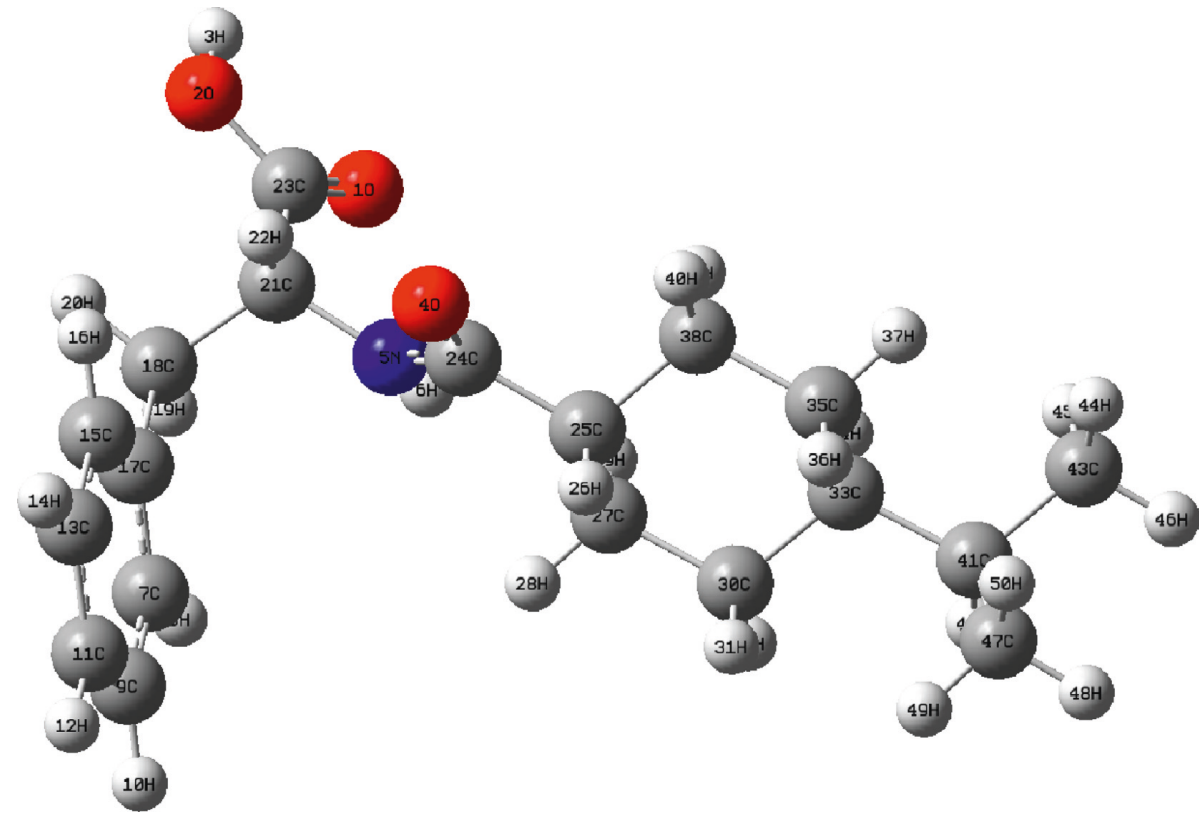

(b)

FIGURE 1: The optimized monomer structures of antidiabetic molecule nateglinide: (a) Monomer 1; (b) Monomer 2. Monomer 1: 6-31 + G(d, p); $E(R B 3 L Y P)=-1020.83682668$ a.u.; dipole moment $=5.1216$ debye; Monomer 2: $6-31+\mathrm{G}(\mathrm{d}, \mathrm{p})$; $\mathrm{E}(\mathrm{RB} 3 \mathrm{LYP})=-1020.83864468$ a.u.; dipole moment $=2.3479$ debye.

DFT/B3LYP/6-31G + (d, p) level. Scaling factors were used for theoretical vibrational wavenumbers. The computed vibrational wavenumbers were scaled as 0.964 for frequencies at the B3LYP/6-31G+ $(d, p)$ basis set [29]. The experimental and simulated IR and Raman spectra of the title compound are given in Figures 2 and 3, respectively.

The characteristic bond of nateglinide was observed at $1647 \mathrm{~cm}^{-1}$ with-C=O functional group, $1715 \mathrm{~cm}^{-1}$ with $-\mathrm{COOH}$ functional group, $2859-3064 \mathrm{~cm}^{-1}$ with $-\mathrm{CH} 2$ functional group, and $3308 \mathrm{~cm}^{-1}$ with $-\mathrm{NH}$ functional group [30]. The $\mathrm{C}=\mathrm{O}$ peak was observed at $1650 \mathrm{~cm}^{-1}$ with an intensity of $333.887 \mathrm{D}\left(10-40 \mathrm{esu}^{2} \cdot \mathrm{cm}^{2}\right)$ [31]. The $\mathrm{C}=\mathrm{O}$ stretching vibration of nateglinide was observed at 1711 (IR), 1647 (IR)-1647 (R) cm ${ }^{-1}$, and 1339 (IR)-1339 (R), and the computed scaled wavenumber values for this band were obtained at $1746 \mathrm{~cm}^{-1}, 1671 \mathrm{~cm}^{-1}$, and $1341 \mathrm{~cm}^{-1}$.

$\mathrm{NH}$ stretching peaks appear at $3585.48 \mathrm{~cm}^{-1}$ and $3710.51 \mathrm{~cm}^{-1}$ [31]. In the present case, $\mathrm{NH}$ stretching modes are calculated at $3508 \mathrm{~cm}^{-1}$.

The peaks at $2921.26 \mathrm{~cm}^{-1}-3147.88 \mathrm{~cm}^{-1}$ range have the highest intensity of $122.03 \mathrm{D}\left(10^{-40} \mathrm{esu}^{2} \cdot \mathrm{cm}^{2}\right)$ due to $\mathrm{C}-\mathrm{H}$ (aromatic) single stretching and $\mathrm{OH}$ sharp peak for the 


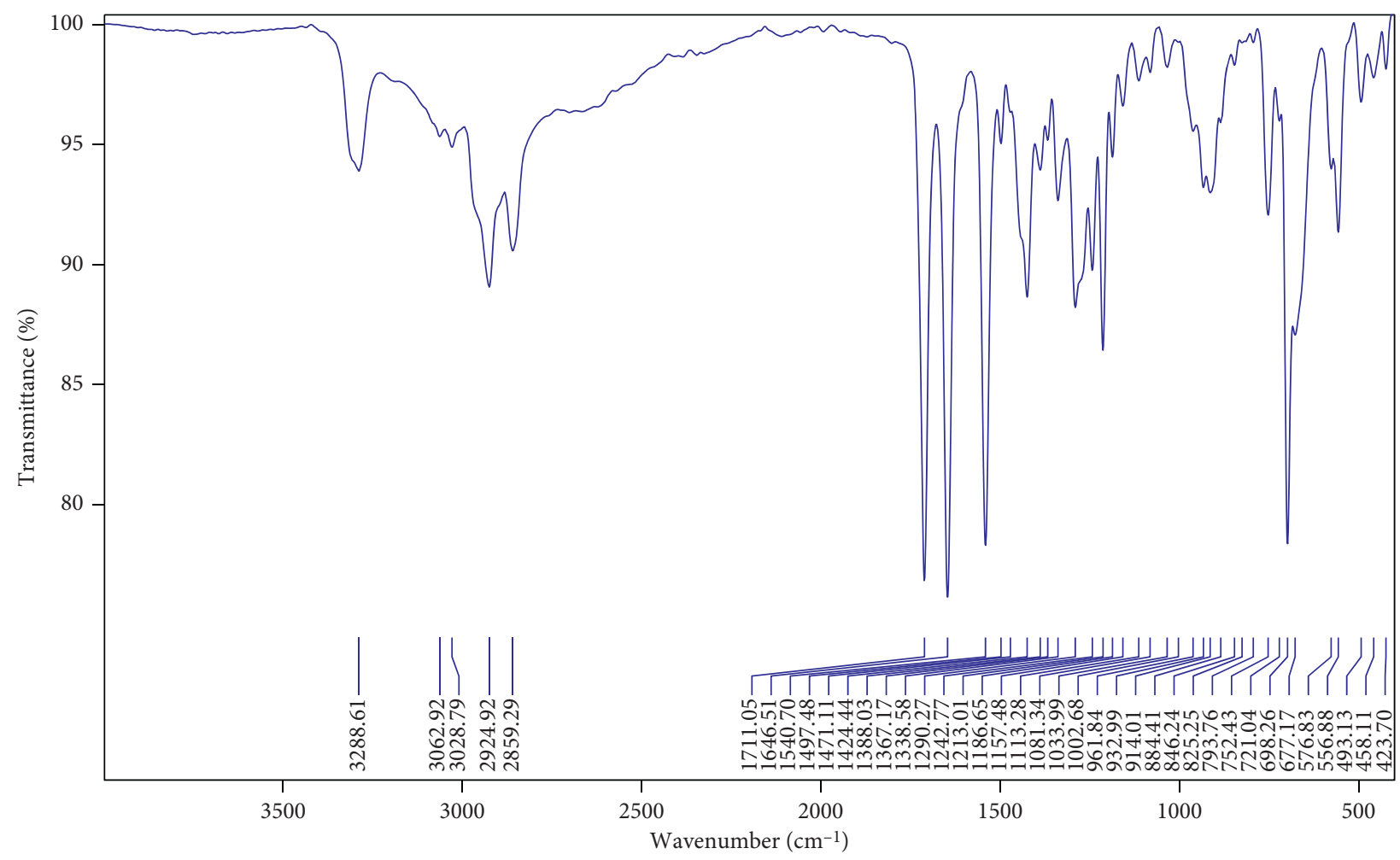

(a)

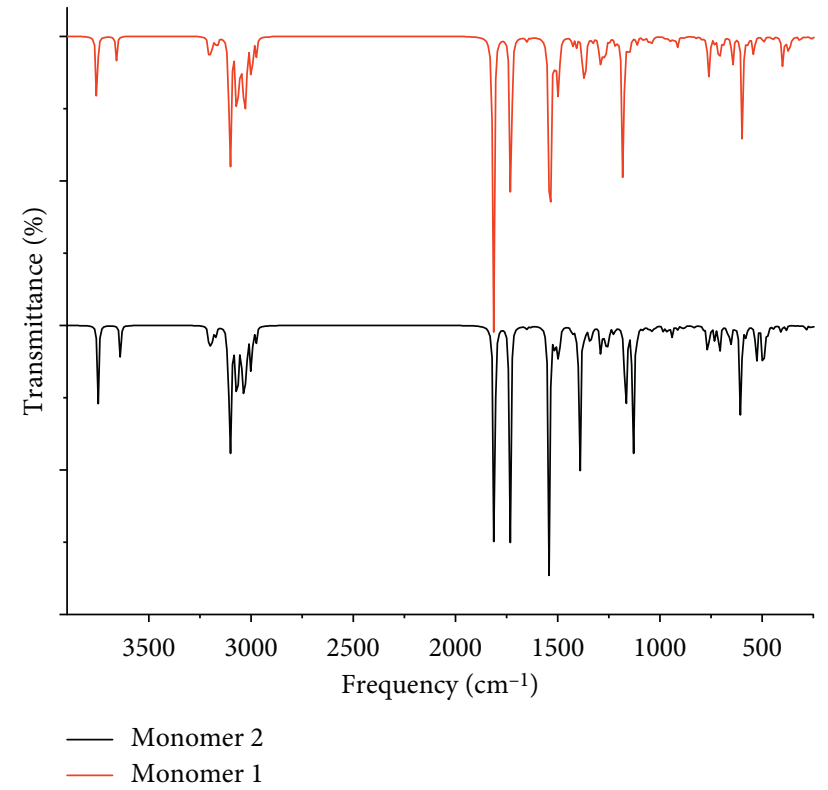

(b)

FIgURE 2: The experimental IR spectrum of nateglinide (a) and simulated IR spectra of nateglinide for Monomer 1 and for Monomer 2 (b).

infrared spectrum. There is strong symmetric stretching between carbon and hydrogen $(\nu \mathrm{CH})$ at $2800-3000 \mathrm{~cm}^{-1}$ frequency range for the Raman spectrum [31]. The $\mathrm{C}-\mathrm{H}$ aromatic stretching vibration and $\mathrm{C}-\mathrm{H}$ aliphatic stretching vibrations are at $3074 \mathrm{~cm}^{-1}, 2933 \mathrm{~cm}^{-1}$, and $2860.88 \mathrm{~cm}^{-1}$ [32]. The $\mathrm{C}-\mathrm{H}$ stretching bands in molecule were observed at $3289,3063 \mathrm{~cm}^{-1}, 3029 \mathrm{~cm}^{-1}, 2925 \mathrm{~cm}^{-1}$, and $2859 \mathrm{~cm}^{-1}$ in the FT-IR spectrum and at $3285 \mathrm{~cm}^{-1}, \quad 3063 \mathrm{~cm}^{-1}$,
$2937 \mathrm{~cm}^{-1}$, and $2860 \mathrm{~cm}^{-1}$ in the Laser-Raman spectrum. These bands were computed at $3091 \mathrm{~cm}^{-1}, 3079.98 \mathrm{~cm}^{-1}$, $3071 \mathrm{~cm}^{-1}, 3062 \mathrm{~cm}^{-1}, 3057 \mathrm{~cm}^{-1}, 2934 \mathrm{~cm}^{-1}, 2922 \mathrm{~cm}^{-1}$, and $2868 \mathrm{~cm}^{-1}$ in our calculations.

The $\mathrm{O}-\mathrm{H}$ stretching band in the title molecule was computed at $3613 \mathrm{~cm}^{-1}$ in the FT-IR spectrum. The $\mathrm{OH}$ inplane bending vibrations $(\delta \mathrm{HOC})$ were experimentally obtained at $1290 \mathrm{~cm}^{-1}$ (IR) (cal. with $15 \%$ contribution of 


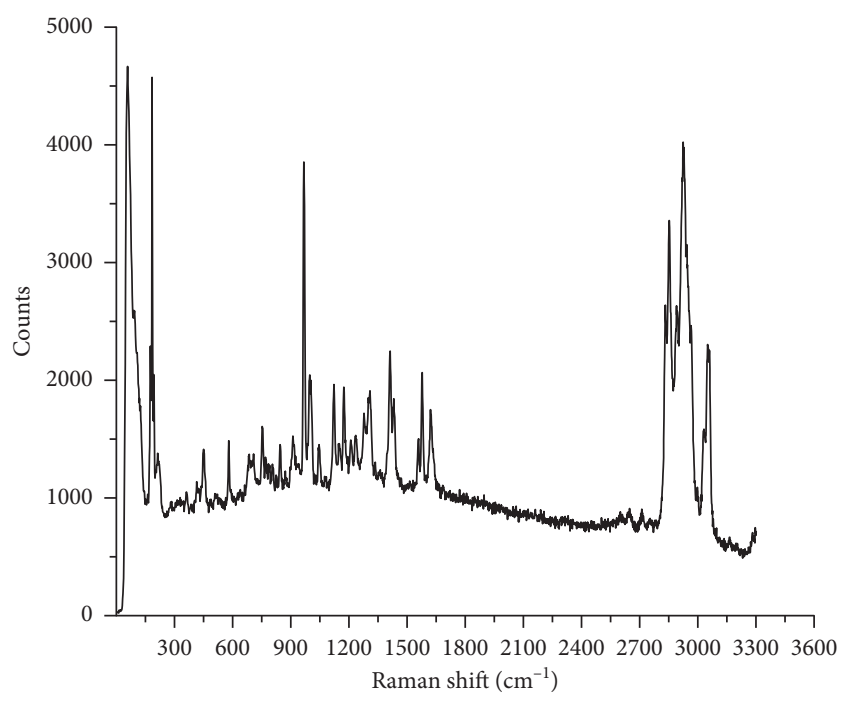

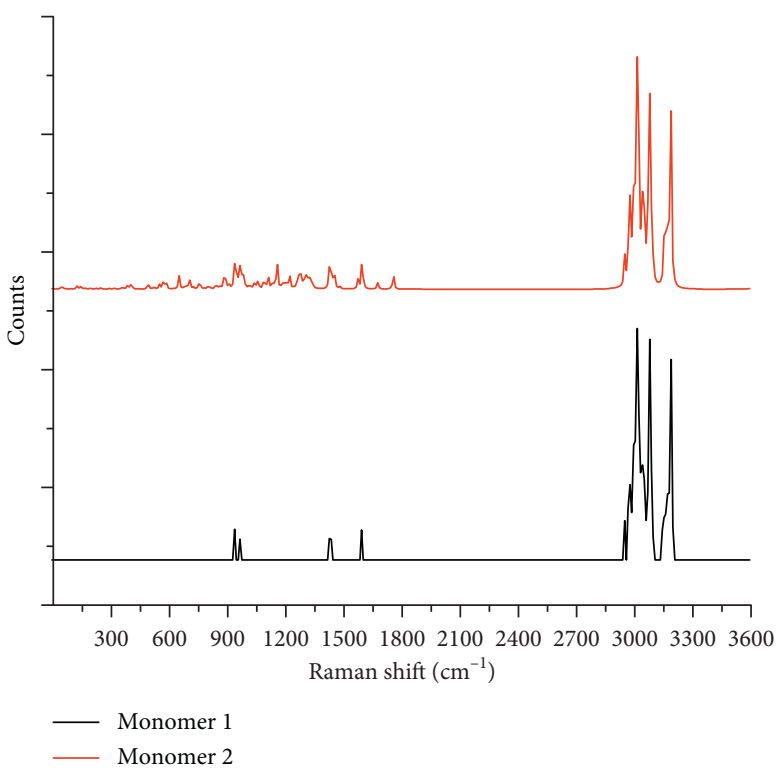

(b)

Figure 3: The experimental Laser-Raman spectrum of nateglinide (a) and simulated Raman spectra of nateglinide for Monomer 1 and for Monomer 2 (b).

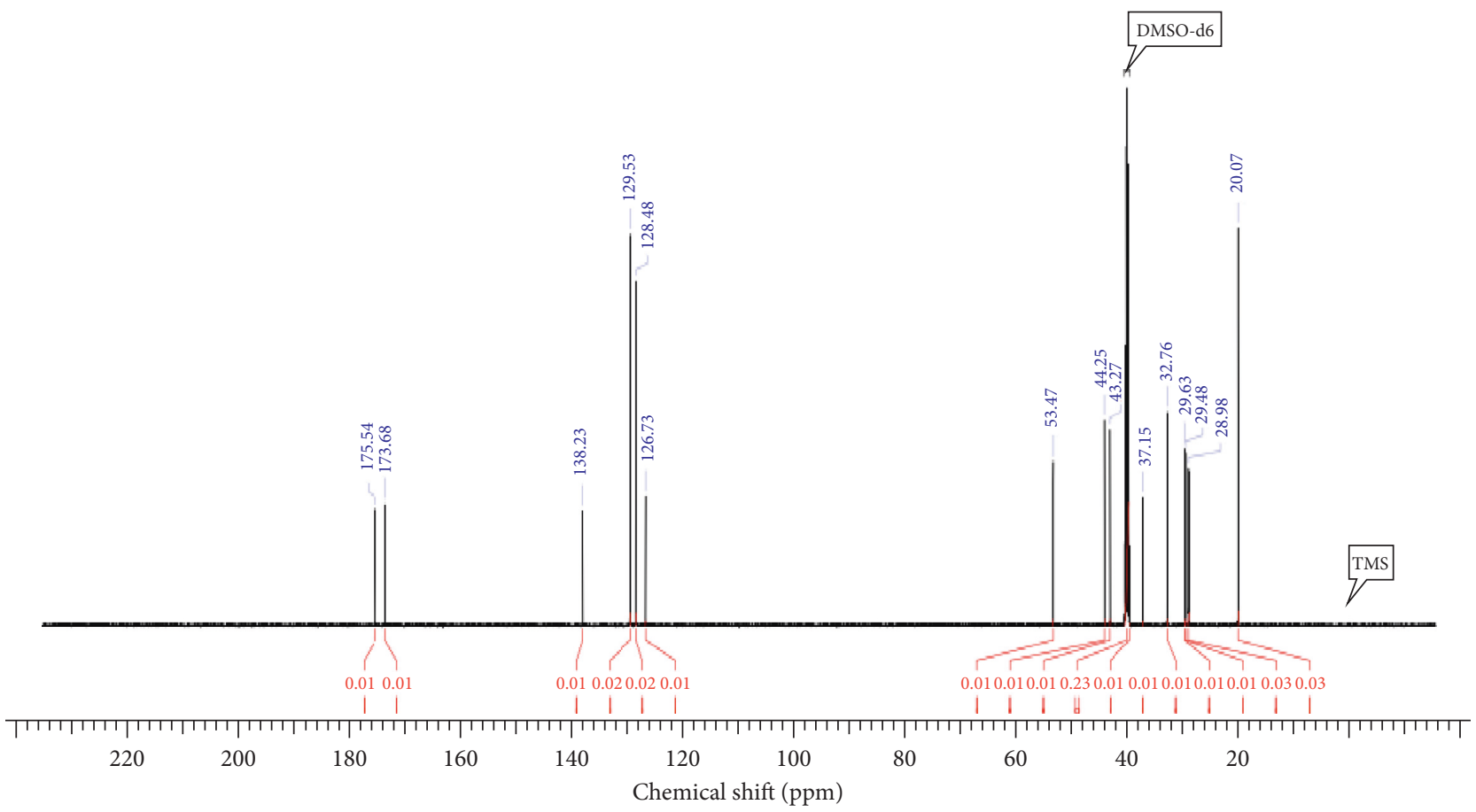

FIgURE 4: The experimental ${ }^{13} \mathrm{C}$ NMR chemical shift spectra of nateglinide.

PED) and 1243(IR)-1243(R) (cal. with 13\% contribution of $\mathrm{PED}) \mathrm{cm}^{-1}$. The $\mathrm{OH}$ in-plane bending vibrations $(\delta \mathrm{HOC})$ were calculated at $1291 \mathrm{~cm}^{-1}$ (IR) and $1230 \mathrm{~cm}^{-1}$ (IR). The $\mathrm{OH}$ out-of-plane bending mode $(\tau \mathrm{HOCC})$ was observed at $1290 \mathrm{~cm}^{-1}$ (IR) and $1187 \mathrm{~cm}^{-1}$ (IR) $-1182 \mathrm{~cm}^{-1}$ (R), whereas it was computed at $1291 \mathrm{~cm}^{-1}$ and $1180 \mathrm{~cm}^{-1}$ with $17 \%$ and $13 \%$ contribution of PED, respectively.
3.3. ${ }^{1} \mathrm{H}$ and ${ }^{13} \mathrm{C} N M R$ Chemical Shift Analyses. The experimental shielding ranges for ${ }^{1} \mathrm{H}$ NMR and ${ }^{13} \mathrm{C}$ NMR are given as $0-13 \mathrm{ppm}$ and $0-180 \mathrm{ppm}$, respectively. ${ }^{1} \mathrm{H}$ and ${ }^{13} \mathrm{C} \mathrm{NMR}$ chemical shift calculated with gauge-including atomic orbital (GIAO) approach using Gaussian 09 software shows good agreement with the experimental chemical shift. Figures 4 and 5 show the experimental ${ }^{1} \mathrm{H}$ and ${ }^{13} \mathrm{C} \mathrm{NMR}$ 


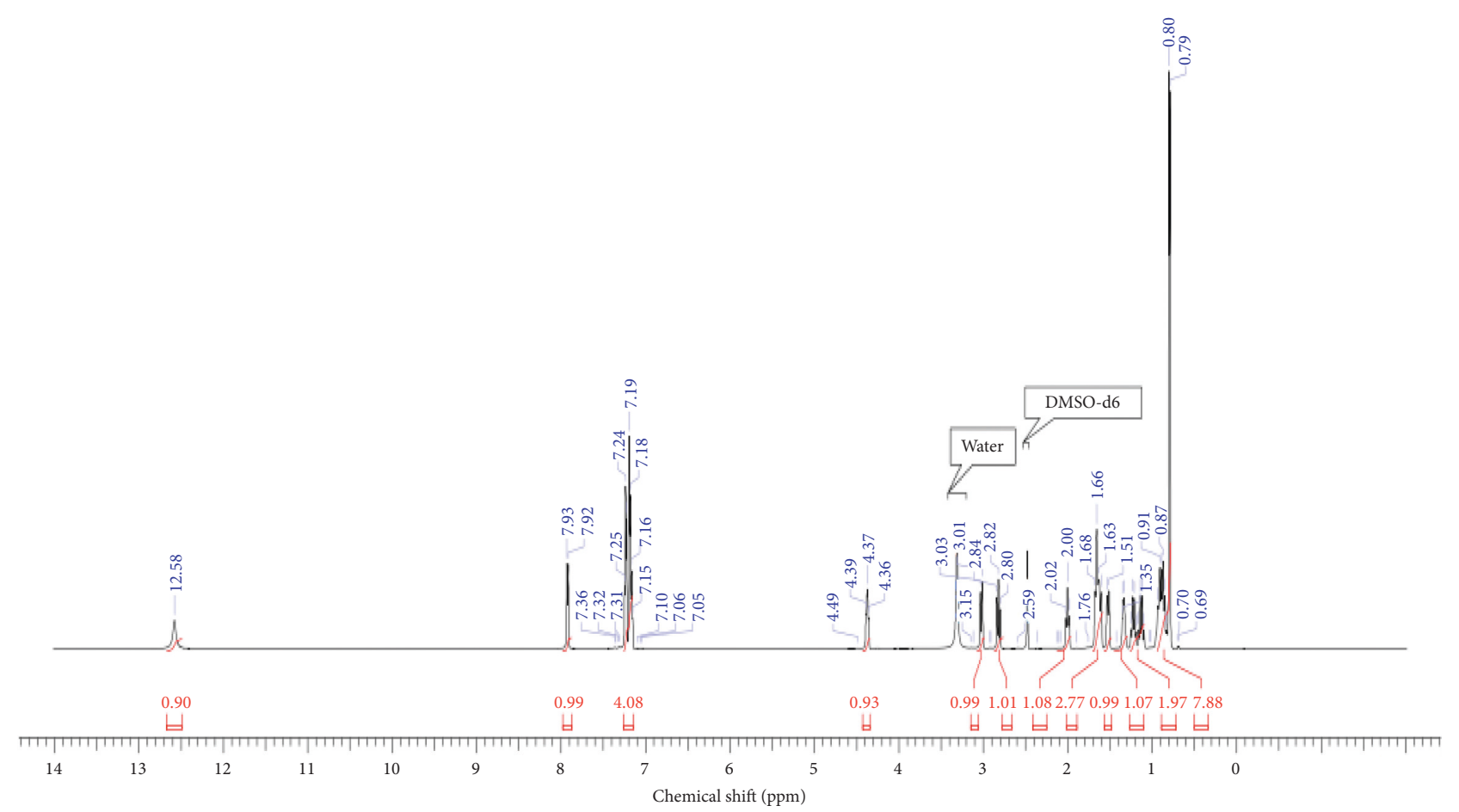

Figure 5: The experimental ${ }^{1} \mathrm{H}$ NMR chemical shift spectra of nateglinide.

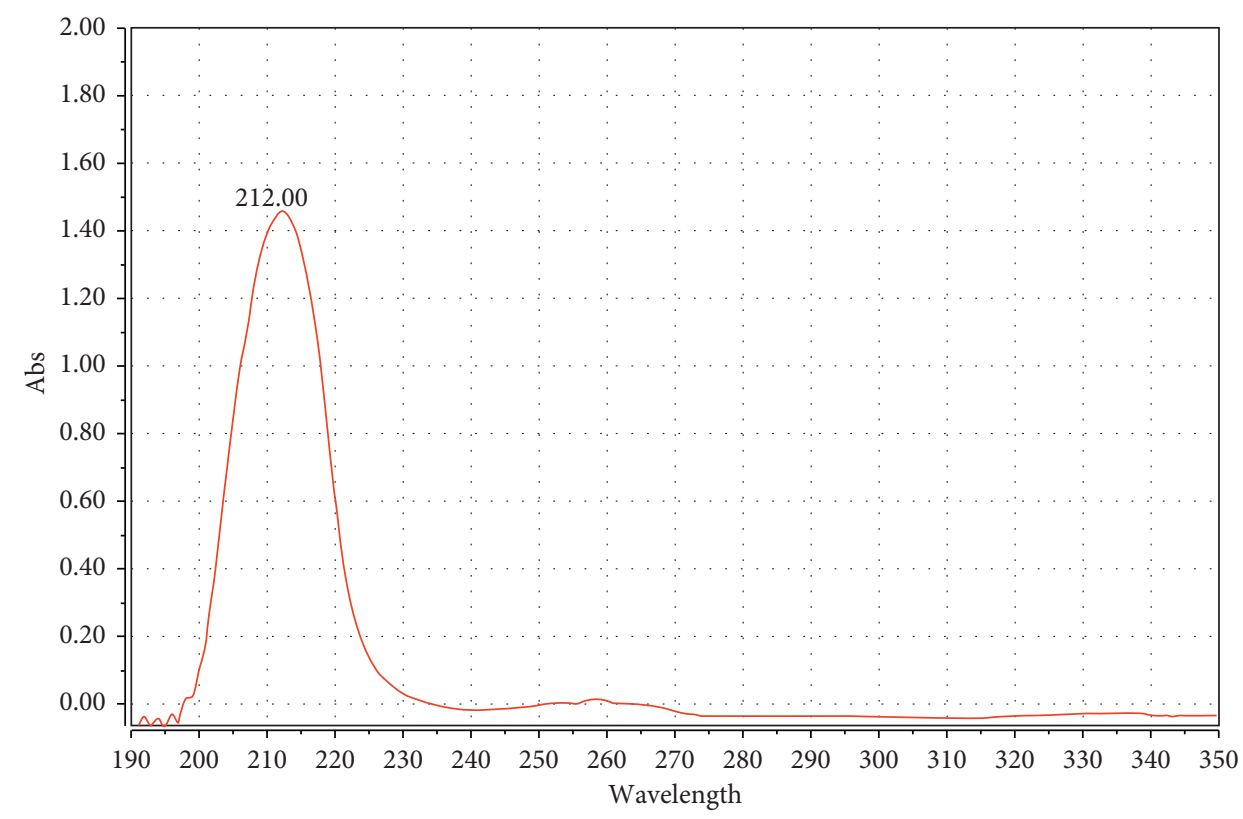

Figure 6: The experimental UV-Vis analysis of antidiabetic molecule nateglinide in methanol.

chemical shift spectra of nateglinide. The experimental ${ }^{1} \mathrm{H}$ and ${ }^{13} \mathrm{C}$ chemical shift values measured in DMSO- $\mathrm{d}_{6}$ solvent and the chemical shift values calculated at the DFT/B3LYP/6-31G $+(\mathrm{d}, \mathrm{p})$ level in DMSO solvent are shown in Tables 5 and 6.

${ }^{1} \mathrm{H}$ chemical shift values for Monomer 1 were computed at the intervals of $0.8078-8.1579 \mathrm{ppm}$ in DMSO. ${ }^{1} \mathrm{H}$ chemical shift values for Monomer 2 were computed at the intervals of 0.8144-8.089 ppm in DMSO. The experimental chemical shifts of ${ }^{1} \mathrm{H}$ are measured in the range of $0.69-12.58 \mathrm{ppm}$. The largest deviation between the calculated and experimental ${ }^{1} \mathrm{H}$ NMR chemical shifts $\left(\delta_{\exp }-\delta_{\text {cal. }}\right)$ was obtained for H16 with $0.6135 \mathrm{ppm}$, whereas the smallest deviation was found for $\mathrm{H} 37$ with $0.0009 \mathrm{ppm}$ for Monomer 1. The largest deviation between the calculated and experimental ${ }^{1} \mathrm{H}$ NMR chemical shifts $\left(\delta_{\exp }-\delta_{\text {cal. }}\right)$ was obtained for $\mathrm{H} 22$ with $1.4 \mathrm{ppm}$, whereas the smallest deviation was found for H34 with $0.0028 \mathrm{ppm}$ for Monomer 2. 
TABLE 1: The experimental and computed vibrational wavenumbers and vibrational assignments of nateglinide results.

\begin{tabular}{|c|c|c|c|c|c|c|}
\hline \multirow{2}{*}{$\begin{array}{l}\text { Assignment (PED\%) } \\
\text { Molecular formula: } \mathrm{C}_{19} \mathrm{H}_{27} \mathrm{NO}_{3}\end{array}$} & \multicolumn{2}{|c|}{ Exp. freq. $\left(\mathrm{cm}^{-1}\right)$} & \multicolumn{4}{|c|}{ The computed parameters for Monomer 2} \\
\hline & IR & Raman & Freq. & Scaled freq. & $I_{\mathrm{IR}}$ & $S_{\text {Raman }}$ \\
\hline$v_{\mathrm{s}} \mathrm{O}_{2} \mathrm{H}_{3}(100)$ & & & 3748 & 3613 & 78.26 & 226.52 \\
\hline$v_{\mathrm{s}} \mathrm{N}_{5} \mathrm{H}_{6}(100)$ & & & 3639 & 3508 & 32.33 & 56.46 \\
\hline$\nu_{\mathrm{s}} \mathrm{C}_{9} \mathrm{H}_{10}(17)+v_{\mathrm{s}} \mathrm{C}_{11} \mathrm{H}_{12}\left(44+v_{\mathrm{s}} \mathrm{C}_{13} \mathrm{H}_{14}(32)\right.$ & 3289 & 3285 & 3206 & 3091 & 15.57 & 292.02 \\
\hline$v_{s} \mathrm{C}_{13} \mathrm{H}_{14}(39)+v_{\mathrm{s}} \mathrm{C}_{15} \mathrm{H}_{16}(10)$ & & & 3195 & 3079,98 & 29.04 & 42.71 \\
\hline$v_{\mathrm{s}} \mathrm{C}_{15} \mathrm{H}_{16}(18)$ & & & 3186 & 3071 & 8.57 & 107.31 \\
\hline$\nu_{s} \mathrm{C}_{13} \mathrm{H}_{14}(22)+v_{s} \mathrm{C}_{15} \mathrm{H}_{16}(67)$ & 3063 & 3063 & 3176 & 3062 & 3.57 & 57.71 \\
\hline$\nu_{\mathrm{S}} \mathrm{C}_{7} \mathrm{H}_{8}(68)+\nu_{\mathrm{s}} \mathrm{C}_{9} \mathrm{H}_{10}(23)$ & 3029 & & 3171 & 3057 & 7.64 & 33.74 \\
\hline$v_{\mathrm{s}} \mathrm{C}_{18} \mathrm{H}_{19}(64)+v_{\mathrm{s}} \mathrm{C}_{18} \mathrm{H}_{20}(35)$ & & 2937 & 3044 & 2934 & 22.81 & 112.46 \\
\hline$v_{s} \mathrm{C}_{43} \mathrm{H}_{44}(28)+v_{s} \mathrm{C}_{43} \mathrm{H}_{45}(32)+v_{s} \mathrm{C}_{43} \mathrm{H}_{46}(28)$ & 2925 & & 3031 & 2922 & 36.09 & 53.34 \\
\hline$v_{\mathrm{s}} \mathrm{C}_{33} \mathrm{H}_{34}(93)$ & 2859 & 2860 & 2975 & 2868 & 16.76 & 44.91 \\
\hline$\nu_{\mathrm{s}} \mathrm{O}_{1} \mathrm{C}_{23}(86)$ & 1711 & & 1811 & 1746 & 279.97 & 16.72 \\
\hline$v_{\mathrm{SO}} \mathrm{C}_{24}(83)$ & 1647 & 1647 & 1733 & 1671 & 224.63 & 6.75 \\
\hline$\nu_{s} \mathrm{C}_{9} \mathrm{C}_{7}(12)+\nu_{\mathrm{s}} \mathrm{C}_{15} \mathrm{C}_{13}(26)$ & & 1607 & 1654 & 1594 & 4.06 & 36.96 \\
\hline$\nu_{s} \mathrm{C}_{11} \mathrm{C}_{9}(29)+\nu_{\mathrm{s}} \mathrm{C}_{17} \mathrm{C}_{7}(22)+\delta \mathrm{C}_{15} \mathrm{C}_{13} \mathrm{C}_{11}(12)$ & 1541 & & 1633 & 1574 & 1.19 & 8.95 \\
\hline$v_{\mathrm{s}} \mathrm{N}_{5} \mathrm{C}_{24}(19)+\delta \mathrm{H}_{6} \mathrm{~N}_{5} \mathrm{C}_{24}(50)$ & 1497 & & 1542 & 1486 & 257.57 & 2.00 \\
\hline $\begin{array}{l}\delta \mathrm{H}_{8} \mathrm{C}_{7} \mathrm{C}_{9}(15)+\delta \mathrm{H}_{10} \mathrm{C}_{9} \mathrm{C}_{11}(18)+\delta \mathrm{H}_{14} \mathrm{C}_{13} \mathrm{C}_{11}(18) \\
+\delta \mathrm{H}_{16} \mathrm{C}_{15} \mathrm{C}_{17}(16)+\delta \mathrm{C}_{13} \mathrm{C}_{11} \mathrm{C}_{9}(10)\end{array}$ & 1471 & & 1534 & 1479 & 10.80 & 0.38 \\
\hline $\begin{array}{l}\delta \mathrm{H}_{44} \mathrm{C}_{43} \mathrm{H}_{46}(20)+\delta \mathrm{H}_{46} \mathrm{C}_{43} \mathrm{H}_{45}(15) \\
+\delta \mathrm{H}_{48} \mathrm{C}_{47} \mathrm{H}_{50}(20)+\delta \mathrm{H}_{49} \mathrm{C}_{47} \mathrm{H}_{48}(24)\end{array}$ & & 1462 & 1513 & 1459 & 8.37 & 10.34 \\
\hline$\delta \mathrm{H}_{32} \mathrm{C}_{30} \mathrm{H}_{31}(29)+\delta \mathrm{H}_{37} \mathrm{C}_{35} \mathrm{H}_{36}(31)$ & & 1440 & 1490 & 1436 & 0.69 & 8.01 \\
\hline$\delta \mathrm{H}_{12} \mathrm{C}_{11} \mathrm{C}_{9}(18)+\delta \mathrm{H}_{20} \mathrm{C}_{18} \mathrm{H}_{19}$ & 1424 & & 1487 & 1433 & 7.34 & 4.42 \\
\hline $\begin{array}{l}\delta \mathrm{H}_{44} \mathrm{C}_{43} \mathrm{H}_{46}(13)+\delta \mathrm{H}_{45} \mathrm{C}_{43} \mathrm{H}_{44}(10)+\delta \mathrm{H}_{48} \mathrm{C}_{47} \mathrm{H}_{50}(17) \\
+\delta \mathrm{H}_{49} \mathrm{C}_{47} \mathrm{H}_{48}(15)+\delta \mathrm{H}_{50} \mathrm{C}_{47} \mathrm{H}_{49}(15)\end{array}$ & 1388 & & 1430 & 1379 & 9.88 & 0.76 \\
\hline$\delta \mathrm{H}_{34} \mathrm{C}_{33} \mathrm{H}_{35}(11)+\delta \mathrm{H}_{45} \mathrm{C}_{43} \mathrm{H}_{44}(11)+\delta \mathrm{H}_{46} \mathrm{C}_{43} \mathrm{H}_{45}(13)$ & 1367 & & 1411 & 1360 & 8.04 & 0.36 \\
\hline$\nu_{\mathrm{s}} \mathrm{O}_{2} \mathrm{C}_{23}(12)+v_{\mathrm{s}} \mathrm{O}_{23} \mathrm{C}_{21}(10)+\delta \mathrm{H}_{22} \mathrm{C}_{21} \mathrm{C}_{23}(23)$ & 1339 & 1339 & 1391 & 1341 & 138.92 & 6.34 \\
\hline $\begin{array}{l}v s \mathrm{C}_{9} \mathrm{C}_{7}(11)+v s \mathrm{C}_{13} \mathrm{C}_{11}(13)+v s \mathrm{C}_{15} \mathrm{C}_{13}(12)+ \\
\delta \mathrm{H}_{8} \mathrm{C}_{7} \mathrm{C}_{9}(16)+\delta \mathrm{H}_{12} \mathrm{C}_{11} \mathrm{C}_{9}(10)+\delta \mathrm{H}_{16} \mathrm{C}_{15} \mathrm{C}_{17}(19)\end{array}$ & & 1311 & 1361 & 1312 & 1.08 & 2.19 \\
\hline $\begin{array}{l}\delta \mathrm{H}_{3} \mathrm{O}_{2} \mathrm{C}_{23}(15)+\tau \mathrm{H}_{20} \mathrm{C}_{18} \mathrm{C}_{17} \mathrm{C}_{15}(24) \\
+\tau \mathrm{H}_{22} \mathrm{C}_{21} \mathrm{C}_{23} \mathrm{O}_{2}(17)\end{array}$ & 1290 & & 1339 & 1291 & 12.17 & 6.90 \\
\hline$\delta \mathrm{H}_{26} \mathrm{C}_{25} \mathrm{C}_{38}(23)$ & & 1268 & 1314 & 1267 & 2.01 & 1.47 \\
\hline$\delta \mathrm{H}_{3} \mathrm{O}_{2} \mathrm{C}_{23}(13)+\delta \mathrm{H}_{22} \mathrm{C}_{21} \mathrm{C}_{23}(28)$ & 1243 & 1243 & 1276 & 1230 & 11.37 & 6.35 \\
\hline$\delta \mathrm{H}_{22} \mathrm{C}_{21} \mathrm{C}_{23}(10)$ & 1213 & 1210 & 1260 & 1215 & 35.96 & 7.82 \\
\hline$\delta \mathrm{H}_{6} \mathrm{~N}_{5} \mathrm{C}_{24}(11)+\delta \mathrm{H}_{19} \mathrm{C}_{18} \mathrm{C}_{17}(17)+\tau \mathrm{H}_{22} \mathrm{C}_{21} \mathrm{C}_{23} \mathrm{O}_{2}(13)$ & 1187 & 1182 & 1224 & 1180 & 10.33 & 12.41 \\
\hline $\begin{array}{l}\nu_{s} \mathrm{C}_{15} \mathrm{C}_{13}(11)+\delta \mathrm{H}_{8} \mathrm{C}_{7} \mathrm{C}_{9}(17)+\delta \mathrm{H}_{10} \mathrm{C}_{9} \mathrm{C}_{11}(16) \\
+\delta \mathrm{H}_{14} \mathrm{C}_{13} \mathrm{C}_{11}(21)+\delta \mathrm{H}_{16} \mathrm{C}_{15} \mathrm{C}_{17}(17)+\end{array}$ & 1157 & 1155 & 1207 & 1164 & 0.71 & 3.24 \\
\hline$\nu_{s} \mathrm{O}_{2} \mathrm{C}_{23}(18)+\nu_{\mathrm{s}} \mathrm{N}_{5} \mathrm{C}_{21}(35)$ & 1113 & & 1131 & 1090 & 150.02 & 7.17 \\
\hline $\begin{array}{l}\nu_{s} \mathrm{C}_{9} \mathrm{C}_{7}(19)+\nu_{\mathrm{s}} \mathrm{C}_{15} \mathrm{C}_{13}(16)+\delta \mathrm{H}_{8} \mathrm{C}_{7} \mathrm{C}_{9}(10) \\
+\delta \mathrm{H}_{12} \mathrm{C}_{11} \mathrm{C}_{9}(12)\end{array}$ & 1081 & 1080 & 1110 & 1070 & 6.74 & 4.44 \\
\hline$\nu \mathrm{sC}_{27} \mathrm{C}_{25}(15)+\nu \mathrm{sC}_{30} \mathrm{C}_{27}(11)+v \mathrm{sC}_{38} \mathrm{C}_{35}(11)$ & 1034 & 1037 & 1089 & 1050 & 1.62 & 3.31 \\
\hline$v_{\mathrm{s}} \mathrm{C}_{38} \mathrm{C}_{35}(20)$ & 1003 & 1003 & 1038 & 1001 & 2.60 & 14.97 \\
\hline $\begin{array}{l}\tau \mathrm{H}_{10} \mathrm{C}_{9} \mathrm{C}_{11} \mathrm{C}_{13}(16)+\tau \mathrm{H}_{12} \mathrm{C}_{11} \mathrm{C}_{9} \mathrm{C}_{7}(31) \\
+\tau \mathrm{H}_{14} \mathrm{C}_{13} \mathrm{C}_{11} \mathrm{C}_{9}(26)+\tau \mathrm{H}_{16} \mathrm{C}_{15} \mathrm{C}_{17} \mathrm{C}_{18}(10)\end{array}$ & 962 & & 1001 & 965 & 0.10 & 0.23 \\
\hline $\begin{array}{l}\tau \mathrm{H}_{8} \mathrm{C}_{7} \mathrm{C}_{9} \mathrm{C}_{11}(14)+\tau \mathrm{H}_{10} \mathrm{C}_{9} \mathrm{C}_{11} \mathrm{C}_{13}(25) \\
+\tau \mathrm{H}_{14} \mathrm{C}_{13} \mathrm{C}_{11} \mathrm{C}_{9}(18)+\tau \mathrm{H}_{16} \mathrm{C}_{15} \mathrm{C}_{17} \mathrm{C}_{18}(18)\end{array}$ & & 948 & 981 & 946 & 0.53 & 0.73 \\
\hline$\nu_{s} \mathrm{C}_{47} \mathrm{C}_{41}(10)+\tau \mathrm{H}_{49} \mathrm{C}_{47} \mathrm{C}_{41} \mathrm{C}_{33}(10)$ & 933 & & 966 & 931 & 4.13 & 6.27 \\
\hline$\tau \mathrm{H}_{16} \mathrm{C}_{15} \mathrm{C}_{17} \mathrm{C}_{18}(10)$ & 914 & 911 & 942 & 908 & 14.03 & 1.89 \\
\hline $\begin{array}{l}\tau \mathrm{H}_{8} \mathrm{C}_{7} \mathrm{C}_{9} \mathrm{C}_{11}(11)+\tau \mathrm{H}_{12} \mathrm{C}_{11} \mathrm{C}_{9} \mathrm{C}_{7}(13) \\
+\tau \mathrm{H}_{16} \mathrm{C}_{15} \mathrm{C}_{17} \mathrm{C}_{18}(10)\end{array}$ & 884 & 880 & 918 & 885 & 0.46 & 3.67 \\
\hline $\begin{array}{l}\tau \mathrm{H}_{8} \mathrm{C}_{7} \mathrm{C}_{9} \mathrm{C}_{11}(26)+\tau \mathrm{H}_{10} \mathrm{C}_{9} \mathrm{C}_{11} \mathrm{C}_{13}(23) \\
+\tau \mathrm{H}_{14} \mathrm{C}_{13} \mathrm{C}_{11} \mathrm{C}_{9}(24)+\tau \mathrm{H}_{16} \mathrm{C}_{15} \mathrm{C}_{17} \mathrm{C}_{18}(26)\end{array}$ & 825 & & 856 & 825 & 0.33 & 0.73 \\
\hline$\delta \mathrm{C}_{13} \mathrm{C}_{11} \mathrm{C}_{9}(23)$ & 794 & 790 & 831 & 801 & 1.83 & 3.28 \\
\hline$\tau \mathrm{C}_{17} \mathrm{C}_{7} \mathrm{C}_{9} \mathrm{C}_{11}(10)$ & 752 & 754 & 766 & 738 & 34.94 & 2.67 \\
\hline$\tau \mathrm{O}_{4} \mathrm{C}_{25} \mathrm{~N}_{5} \mathrm{C}_{24}(25)+\tau \mathrm{O}_{1} \mathrm{C}_{21} \mathrm{O}_{2} \mathrm{C}_{23}(10)$ & 721 & 720 & 757 & 730 & 9.88 & 1.25 \\
\hline$\nu_{s} \mathrm{C}_{21} \mathrm{C}_{18}(10)+\delta \mathrm{C}_{13} \mathrm{C}_{11} \mathrm{C}_{9}(10)+\tau \mathrm{O}_{1} \mathrm{C}_{21} \mathrm{O}_{2} \mathrm{C}_{23}(36)$ & 698 & & 733 & 707 & 13.36 & 10.82 \\
\hline $\begin{array}{l}\tau \mathrm{H}_{8} \mathrm{C}_{7} \mathrm{C}_{9} \mathrm{C}_{11}(13)+\tau \mathrm{C}_{13} \mathrm{C}_{11} \mathrm{C}_{9} \mathrm{C}_{7}(13) \\
+\tau \mathrm{C}_{15} \mathrm{C}_{13} \mathrm{C}_{11} \mathrm{C}_{9}(19)+\tau \mathrm{C}_{17} \mathrm{C}_{7} \mathrm{C}_{9} \mathrm{C}_{11}(22)\end{array}$ & 677 & & 709 & 683 & 39.63 & 0.27 \\
\hline$\delta \mathrm{O}_{1} \mathrm{C}_{23} \mathrm{O}_{2}(41)$ & 577 & 625 & 655 & 631 & 29.30 & 8.83 \\
\hline$\delta \mathrm{C}_{17} \mathrm{C}_{7} \mathrm{C}_{9}(11)$ & 557 & & 580 & 559 & 8.75 & 2.77 \\
\hline$\gamma \mathrm{C}_{41} \mathrm{C}_{30} \mathrm{C}_{35} \mathrm{C}_{33}(12)$ & 458 & 458 & 472 & 455 & 7.50 & 2.39 \\
\hline
\end{tabular}


TABle 1: Continued.

\begin{tabular}{|c|c|c|c|c|c|c|}
\hline \multirow{2}{*}{$\begin{array}{l}\text { Assignment (PED\%) } \\
\text { Molecular formula: } \mathrm{C}_{19} \mathrm{H}_{27} \mathrm{NO}_{3}\end{array}$} & \multicolumn{2}{|c|}{ Exp. freq. $\left(\mathrm{cm}^{-1}\right)$} & \multicolumn{4}{|c|}{ The computed parameters for Monomer 2} \\
\hline & IR & Raman & Freq. & Scaled freq. & $I_{\mathrm{IR}}$ & $S_{\text {Raman }}$ \\
\hline$\delta \mathrm{N}_{5} \mathrm{C}_{21} \mathrm{C}_{18}(10)$ & 424 & & 444 & 428 & 2.74 & 0.98 \\
\hline $\begin{array}{l}\tau \mathrm{H}_{8} \mathrm{C}_{7} \mathrm{C}_{9} \mathrm{C}_{11}(12)+\tau \mathrm{C}_{13} \mathrm{C}_{11} \mathrm{C}_{9} \mathrm{C}_{7}(19) \\
+\tau \mathrm{C}_{15} \mathrm{C}_{13} \mathrm{C}_{11} \mathrm{C}_{9}(36)\end{array}$ & & 406 & 414 & 399 & 0.11 & 0.03 \\
\hline$\delta \mathrm{O}_{2} \mathrm{C}_{23} \mathrm{C}_{21}(14)$ & & 261 & 286 & 276 & 5.95 & 0.70 \\
\hline$\tau \mathrm{C}_{38} \mathrm{C}_{35} \mathrm{C}_{33} \mathrm{C}_{30}(12)$ & & 227 & 241 & 232 & 1.00 & 0.31 \\
\hline
\end{tabular}

s, symmetric; as, asymmetric; $\boldsymbol{v}$, stretching; $\boldsymbol{\delta}$, in-plane bending; $\tau$, torsion; $\gamma$, out-of-plane bending; $d_{\mathrm{s}}$, scissoring and symmetric bending; $\rho$, rocking; $t$, twisting; $w$, wagging; $I_{\mathrm{IR}}$, IR intensity $(\mathrm{km} / \mathrm{mol}) ; S_{\text {Raman, }}$ Raman scattering activity; PED, potential energy distribution; $W$, wavenumber $\left(\mathrm{cm}^{-1}\right) ; T$, transmittance (\%). $R^{2}=0.9981$ for Monomer 1 and $R^{2}=0.9980$ for Monomer 2 for IR wavenumbers.

TABLE 2: The experimental and computed UV-Vis parameters and electronic transitions in methanol of nateglinide.

\begin{tabular}{|c|c|c|c|c|c|c|}
\hline $\begin{array}{l}\text { The experimental } \\
\text { parameters }\end{array}$ & $\begin{array}{c}\text { Calculated parameter for } \\
\text { Monomer } 2\end{array}$ & The ca & $\begin{array}{l}\text { lculated } \\
\mathrm{crf}=(\mathrm{cp}\end{array}$ & $\begin{array}{l}\text { arameters for } \\
\text { 1, solvent }=\mathrm{me}\end{array}$ & $\begin{array}{l}\text { nomer } 1(t \\
\text { nol) maxdi }\end{array}$ & $\begin{array}{l}=(\text { nstates }=6) B 3 L Y P / 6-31+\mathrm{G}(\mathrm{d}, \mathrm{p}) \\
k=22 \mathrm{~GB} \text { geom }=\text { connectivity })\end{array}$ \\
\hline$\lambda_{\exp }(\mathrm{nm})$ & Transitions & $\begin{array}{l}\lambda_{\mathrm{cal}} \\
(\mathrm{nm})\end{array}$ & $\begin{array}{l}\lambda_{\mathrm{cal}} \\
(\mathrm{nm})\end{array}$ & $\begin{array}{l}\text { Excitation } \\
\text { energy }(\mathrm{eV})\end{array}$ & $\begin{array}{l}\text { Oscillator } \\
\text { strength }\end{array}$ & Major contributions [31] \\
\hline \multirow{6}{*}{212} & $n \longrightarrow \sigma^{*}$ & 236.49 & 236.51 & 5.2421 & 0.0046 & $\begin{array}{c}\text { H-1->LUMO }(35 \%), \text { HOMO->L + } 1 \\
(41 \%) \text { H-2->LUMO }(3 \%), \text { H-1->L } \\
+1(4 \%), \text { H-1->L + } 2(5 \%), \text { HOMO- } \\
>\text { LUMO }(9 \%)\end{array}$ \\
\hline & $n \longrightarrow \sigma^{*}$ & 228.64 & 228.14 & 5.4345 & 0.0144 & $\begin{array}{c}\text { H-3->LUMO (50\%), H-3->L + } 2 \\
(18 \%) \text { H-7->LUMO (2\%), H-7->L } \\
+2(2 \%), \text { H-6->LUMO (3\%), H-6- } \\
>\text { L + } 2(3 \%), \text { H-1->LUMO }(4 \%) \\
\text { HOMO->LUMO }(7 \%)\end{array}$ \\
\hline & $n \longrightarrow \sigma^{*}$ & 219.7 & 221.79 & 5.5902 & 0.0065 & $\begin{array}{c}\mathrm{H}-2->\mathrm{L}+2(25 \%), \mathrm{H}-2->\mathrm{L}+4(39 \%) \\
\mathrm{H}-2->\mathrm{LUMO}(3 \%), \mathrm{H}-2->\mathrm{L}+3(5 \%) \\
\mathrm{H}-2->\mathrm{L}+5(6 \%), \mathrm{H}-1->\mathrm{L}+2(4 \%) \\
\mathrm{H}-1->\mathrm{L}+4(5 \%)\end{array}$ \\
\hline & $n \longrightarrow \sigma^{*}$ & 216.37 & 219.16 & 5.6571 & 0.0741 & $\begin{array}{c}\text { HOMO->LUMO (66\%) H-3- } \\
\text { >LUMO (6\%), H-2->LUMO (6\%), } \\
\text { H-2->L+4 } 4(2 \%), \text { H-1->L + } 1(5 \%) \\
\text { HOMO->L+ } 1(6 \%), \text { HOMO->L + } 2 \\
(3 \%)\end{array}$ \\
\hline & $n \longrightarrow \sigma^{*}$ & 212.63 & 216.85 & 5.7175 & 0.0120 & $\begin{array}{c}\text { H-2->LUMO (67\%), H-1->LUMO } \\
(12 \%) \text { H-3->LUMO }(6 \%), \text { H-2->L } \\
+4(5 \%), \text { HOMO->LUMO }(2 \%) \\
\text { H-1->LUMO }(13 \%), \text { H-1->L }+1\end{array}$ \\
\hline & $n \longrightarrow \sigma^{*}$ & 210.78 & 207.28 & 5.9814 & 0.0257 & $\begin{array}{l}(18 \%), \text { HOMO->L + } 2(50 \%) \text { H-2- } \\
>\text { LUMO }(4 \%), H-1->\mathrm{L}+2(6 \%)\end{array}$ \\
\hline
\end{tabular}

The ${ }^{13} \mathrm{C}$ chemical shifts for Monomer 1 were calculated in the range of 7.0408-163.551 ppm in DMSO ppm, and the ${ }^{13} \mathrm{C}$ chemical shifts for Monomer 2 were calculated in the range of 6.1516-161.592 ppm, while they were experimentally recorded in the range of $20.07-175.54 \mathrm{ppm}$. The largest deviation between the calculated and experimental ${ }^{13} \mathrm{C}$ NMR chemical shifts $\left(d_{\exp }-d_{\text {cal. }}\right)$ was obtained for C17 with $14.022 \mathrm{ppm}$, whereas the smallest deviation was found for C33 with $0.6225 \mathrm{ppm}$ for Monomer 1. The largest deviation between the calculated and experimental ${ }^{13} \mathrm{C}$ NMR chemical shifts $\left(d_{\mathrm{exp}}-d_{\text {cal. }}\right)$ was obtained for C23 with $13.948 \mathrm{ppm}$, whereas the smallest deviation was found for C33 with $0.4279 \mathrm{ppm}$ for Monomer 2.
3.4. UV-Vis Analyses. The obtained and simulated UV-Vis spectrum of nateglinide dissolved in methanol was recorded in the region of $190-350 \mathrm{~nm}$. UV-Vis calculation was performed in methanol using the TD-DFT method with Gaussian 09W software and GaussView5 molecular visualization program. The measured and simulated UV-Vis electronic absorption spectra are given in Figures 6 and 7. Additionally, the experimental and computed electronic absorption wavelengths, electronic transitions, oscillator strengths, excitation energies, and major contributions are listed Table 2. Rajasekaran et al. determined a method in which the absorbance of pure drug and tablet extract in $95 \%$ ethanol was measured at $210 \mathrm{~nm}$ [33]. Xavier studied the UV 
TABLE 3: The optimized molecular geometric parameters of nateglinide.

\begin{tabular}{|c|c|c|c|c|c|c|c|c|c|c|c|}
\hline $\begin{array}{l}\text { Bond } \\
\text { lengths }(\AA)\end{array}$ & $\begin{array}{c}\text { X-ray } \\
{[27]}\end{array}$ & Values & $\begin{array}{c}\text { Bond } \\
\text { lengths }(\AA)\end{array}$ & $\begin{array}{c}\text { X-ray } \\
{[27]}\end{array}$ & Values & $\begin{array}{c}\text { Bond } \\
\text { angles }\left({ }^{\circ}\right)\end{array}$ & Values & $\begin{array}{c}\text { X-ray } \\
{[27]}\end{array}$ & $\begin{array}{c}\text { Bond } \\
\text { angles }\left({ }^{\circ}\right)\end{array}$ & Values & $\begin{array}{c}\text { X-ray } \\
{[27]}\end{array}$ \\
\hline C11-C13 & 1.352 & 1.398 & C24-C25 & 1.495 & 1.530 & C11-C13-H14 & 120.102 & 119.116 & C25-C38-C35 & 111.408 & 110.700 \\
\hline $\mathrm{C} 13-\mathrm{C} 15$ & 1.413 & 1.395 & C25-C38 & 1.537 & 1.546 & C15-C13-H14 & 119.705 & 119.160 & C38-C35-C33 & 112.186 & 112.617 \\
\hline C9-C11 & 1.360 & 1.396 & C35-C38 & 1.524 & 1.537 & C9-C11-H12 & 120.254 & 120.606 & C35-C33-C30 & 109.752 & 110.312 \\
\hline C7-C9 & 1.404 & 1.398 & C33-C35 & 1.527 & 1.542 & C13-C11-H12 & 120.199 & 120.467 & C33-C30-C27 & 112.163 & 112.776 \\
\hline C7-C17 & 1.374 & 1.401 & C33-C30 & 1.522 & 1.542 & C13-C15-H16 & 119.437 & 120.306 & $\mathrm{H} 28-\mathrm{C} 27-\mathrm{H} 29$ & & 108.188 \\
\hline C15-C17 & 1.392 & 1.403 & $\mathrm{C} 27-$ & 1.5 & & C11-C9-H10 & 120. & 119. & C27-C25-H26 & & 106.800 \\
\hline C17- & 1.524 & 1.514 & $\mathrm{C} 25$ & & & 15-H16 & 119.745 & 120.288 & $3-\mathrm{H} 40$ & .036 & 108.165 \\
\hline $\mathrm{C} 18-\mathrm{C} 21$ & 1.526 & 1.558 & $\mathrm{C} 33$ & 1.542 & & C7-C9-H10 & 119.774 & 119.650 & C35-C33-H34 & & 106.334 \\
\hline $\mathrm{C} 21-\mathrm{C} 23$ & 1.522 & 1.525 & $\mathrm{C} 41-\mathrm{C} 47$ & 1.493 & & C17-C7-H8 & 119.457 & 119.513 & H37-C35-H36 & & 107.896 \\
\hline $\mathrm{C} 23=\mathrm{O} 1$ & 1.231 & & C41- & & & $\mathrm{C} 17-\mathrm{C}$ & & & $0-\mathrm{H} 32$ & & 107.730 \\
\hline $\mathrm{C} 2$ & 1.308 & 1.353 & $\mathrm{C} 24=$ & 1.252 & 1.230 & H19 & 109.619 & 108.510 & $\mathrm{H} 42$ & 779 & 106.849 \\
\hline & 0.909 & 0.973 & N5-H6 & 0.927 & 1.010 & C21-C18-H19 & 108.662 & 108.488 & $\mathrm{H} 44-\mathrm{C} 43-\mathrm{H} 45$ & & 109.495 \\
\hline C21-N5 & 1.460 & 1.447 & $\mathrm{C} 24=\mathrm{N} 5$ & 1.332 & 1.372 & $\mathrm{C} 21-\mathrm{C} 18-\mathrm{H} 20$ & & 108.534 & C41-C43-H46 & & 109.459 \\
\hline \multicolumn{4}{|c|}{ Dihedral angles $\left({ }^{\circ}\right)$} & & & $\mathrm{C} 23-\mathrm{C} 21-\mathrm{H} 22$ & & & $\mathrm{H} 42-\mathrm{C} 41-\mathrm{C} 47$ & & 106.767 \\
\hline \multicolumn{2}{|c|}{$\mathrm{H} 10-\mathrm{C} 9-\mathrm{C} 7-\mathrm{C} 17$} & \multicolumn{2}{|r|}{179.820} & 179.844 & & N5-C21-H22 & 106.696 & 107.947 & $\mathrm{C} 41-\mathrm{C} 47-\mathrm{H} 50$ & 111.618 & 109.518 \\
\hline \multicolumn{2}{|c|}{ H10-C9-C11-C13 } & \multicolumn{2}{|c|}{-179.816} & 178.624 & & C24-N5-H6 & 119.107 & 121.393 & $\mathrm{H} 49-\mathrm{C} 47-\mathrm{H} 48$ & 107.337 & 109.401 \\
\hline \multicolumn{2}{|c|}{ H16-C15-C13-C11 } & \multicolumn{2}{|c|}{-179.772} & 178.229 & & $\mathrm{C} 23-\mathrm{O} 2-\mathrm{H} 3$ & 107.493 & 106.777 & $\mathrm{C} 25-\mathrm{C} 24-\mathrm{O} 4$ & 120.642 & 121.73 \\
\hline \multicolumn{2}{|c|}{ C9-C7-C17-C18 } & \multicolumn{2}{|c|}{-179.489} & -179.882 & & O4-C24-N5 & & & $\mathrm{C} 24-\mathrm{C} 25-\mathrm{C} 27$ & 117.171 & 116.594 \\
\hline \multicolumn{2}{|c|}{$\mathrm{C} 21-\mathrm{C} 23-\mathrm{O} 2-\mathrm{H} 3$} & \multicolumn{2}{|c|}{-177.502} & 178.064 & & $\mathrm{O} 1-\mathrm{C} 23-\mathrm{O} 2$ & 122.883 & 124.478 & C24-C25-C38 & 109.582 & 109.451 \\
\hline \multicolumn{2}{|c|}{ C21-N5-C24-C25 } & \multicolumn{2}{|c|}{-174.757} & -170.842 & & N5-C24-C25 & 117.375 & 118.654 & C25-C27-C30 & 110.933 & 109.665 \\
\hline \multicolumn{2}{|c|}{ C25-C38-C35-H36 } & \multicolumn{2}{|c|}{-65.703} & -65.679 & & & & & & & \\
\hline
\end{tabular}

[27] L. Tessler, I. Goldberg., bis(nateglinide) hydronium chloride, and its unique self-assembly into extended polymeric arrays via $\mathrm{O}-\mathrm{H} \cdots \mathrm{O}, \mathrm{N}-\mathrm{H} \cdots \mathrm{Cl}$, and $\mathrm{O}-\mathrm{H} \cdots \mathrm{Cl}$ hydrogen bonds. Acta Cryst. C61 (2005) 738-740.

TABLE 4: Zero point, relative energy and dipole moment of nateglinide.

\begin{tabular}{lccc}
\hline Conformers & Zero-point energy (Hartree/particle) & Relative energy (kcal/mole) & Dipole moment (debye) \\
\hline Monomer 1 & -1020.402782 & \multirow{2}{*}{0.99582} & 5.1216 \\
Monomer 2 & -1020.404370 & 2.3479 \\
\hline
\end{tabular}

TABle 5: The experimental and computed ${ }^{1} \mathrm{H}$ NMR isotropic chemical shifts (with respect to TMS, all values in ppm) of nateglinide.

\begin{tabular}{|c|c|c|c|c|c|}
\hline $\begin{array}{l}\delta_{\exp } \\
\left(\text { in DMSO- } d_{6}\right)\end{array}$ & $\begin{array}{l}\text { Monomer } 1 \delta_{\text {cal }} \\
\text { (in DMSO) }\end{array}$ & $\begin{array}{l}\text { Monomer } 2 \delta_{\text {cal }} \\
\quad \text { (in DMSO) }\end{array}$ & $\begin{array}{c}\delta_{\exp } \\
\text { (in } \mathrm{DMSO}-\mathrm{d}_{6} \text { ) }\end{array}$ & $\begin{array}{l}\text { Manomer } 1 \delta_{\text {cal }} \\
\text { (in DMSO) }\end{array}$ & $\begin{array}{l}\text { Manomer } 2 \delta_{\text {cal }} \\
\text { (in DMSO) }\end{array}$ \\
\hline \multirow[t]{3}{*}{12.58} & & & 1.76 & $1.78-\mathrm{H} 42$ & $1.88-\mathrm{H} 37$ \\
\hline & 8.16-H8 & 8.09-H16 & 1.68 & 1.67-H31 & $1.83-\mathrm{H} 26$ \\
\hline & $8.07-\mathrm{H} 10$ & 7.89-H10 & 1.66 & $1.67-\mathrm{H} 28$ & $1.65-\mathrm{H} 32$ \\
\hline $7.93-7.92$ & 7.95-H14 & 7.84-H14 & 1.63 & 1.61-H34 & $1.55-\mathrm{H} 29$ \\
\hline $7.24-7.36$ & 7.83-H12 & 7.78-H12 & 1.51 & $1.54-\mathrm{H} 29$ & $1.51-\mathrm{H} 34$ \\
\hline $7.15-7.19$ & 7.80-H16 & 7.70-H8 & & $1.50-\mathrm{H} 32$ & $1.50-\mathrm{H} 31$ \\
\hline \multirow[t]{2}{*}{$7.05-7.10$} & $6.92-\mathrm{H} 3$ & $7.10-\mathrm{H} 3$ & & $1.49-\mathrm{H} 39$ & $1.31-\mathrm{H} 39$ \\
\hline & 6.16-H6 & $6.20-\mathrm{H} 6$ & 1.35 & 1.35-H37 & $1.23-\mathrm{H} 44$ \\
\hline $4.36-4.49$ & $4.55-\mathrm{H} 22$ & $5.76-\mathrm{H} 22$ & & $1.25-\mathrm{H} 44$ & $1.23-\mathrm{H} 36$ \\
\hline $3.01-3.15$ & 3.48-H19 & $3.71-\mathrm{H} 20$ & & 1.19-H46 & $1.21-\mathrm{H} 49$ \\
\hline $2.80-2.84$ & $2.93-\mathrm{H} 20$ & 2.67-H19 & & $1.13-\mathrm{H} 49$ & 1.19-H46 \\
\hline 2.59 & $2.17-\mathrm{H} 26$ & $2.05-\mathrm{H} 40$ & & $1.10-\mathrm{H} 45$ & $1.08-\mathrm{H} 45$ \\
\hline \multirow[t]{2}{*}{$2.00-2.02$} & $2.15-\mathrm{H} 40$ & 1.94-H42 & $0.91,0.87$ & $0.86-\mathrm{H} 48$ & $0.96-\mathrm{H} 48$ \\
\hline & $1.97-\mathrm{H} 36$ & $1.88-\mathrm{H} 28$ & $0.69,0.70$ & $0.81-\mathrm{H} 50$ & 0.81-H50 \\
\hline
\end{tabular}

$R^{2}=0.9932$ and $\mathrm{RMSD}=0.237831578 \mathrm{ppm}$ for Monomer 1 and $R^{2}=0.9709$ and $\mathrm{RMSD}=0.403748 \mathrm{ppm}$ for Monomer 2.

detector response of NTG and found the best result at $210 \mathrm{~nm}$ [19]. In this research, the wavelength recorded at $212 \mathrm{~nm}$ in the experimental UV-Vis spectrum can be assigned to $\mathrm{n} \longrightarrow \sigma *$ transition. The calculated wavelength corresponding to this experimental value was obtained at $216.85 \mathrm{~nm}$ with $5.7175 \mathrm{eV}$ value of excitation energy and
0.0120 value of oscillator strength for Monomer 1 . The other computed wavelengths are given as $236.51 \mathrm{~nm}, 228.14 \mathrm{~nm}$, $221.79 \mathrm{~nm}, 219.16 \mathrm{~nm}, 216.85 \mathrm{~nm}$, and $207.28 \mathrm{~nm}$. The experimental and computed wavelengths and electronic transitions are in good harmony. The calculated wavelength corresponding to this experimental value was obtained at 
TABLe 6: The experimental and computed ${ }^{13} \mathrm{C}$ NMR isotropic chemical shifts (with respect to TMS, all values in ppm) of nateglinide.

\begin{tabular}{|c|c|c|c|c|c|}
\hline $\begin{array}{l}\delta_{\exp } \\
\left(\text { in DMSO- } \mathrm{d}_{6}\right) \\
\end{array}$ & $\begin{array}{l}\text { Monomer } 1 \delta_{\text {cal }} \\
(\text { in DMSO) }\end{array}$ & $\begin{array}{l}\text { Monomer } 2 \delta_{\text {cal }} \\
\text { (in DMSO) }\end{array}$ & $\begin{array}{c}\delta_{\exp } \\
\text { (in } \mathrm{DMSO}-\mathrm{d}_{6} \text { ) }\end{array}$ & $\begin{array}{c}\delta_{\mathrm{cal}} \\
(\text { in } \mathrm{DMSO})\end{array}$ & $\begin{array}{l}\text { Monomer } 2 \delta_{\text {cal }} \\
\text { (in DMSO) }\end{array}$ \\
\hline 175.54 & 163.551-C24 & $161.592-\mathrm{C} 23$ & $43.27,44.25$ & $39.6492-\mathrm{C} 25$ & $39.5256-\mathrm{C} 25$ \\
\hline 173.68 & 161.646-C23 & 161.179-C24 & 37.15 & $36.5275-\mathrm{C} 33$ & $36.7221-\mathrm{C} 33$ \\
\hline 138.23 & 124.208-C17 & 124.354-C17 & 32.76 & 30.134-C18 & $33.2255-\mathrm{C} 18$ \\
\hline 129.53 & 115.937-C9 & 116.639-C7 & 29.63 & 27.5208-C41 & 27.5424-C41 \\
\hline 128.48 & 115.795-C15 & 115.406-C15 & 29.48 & 25.2552-C38 & 25.943-C38 \\
\hline \multirow[t]{3}{*}{126.73} & 115.334-C13 & 114.909-C9 & 28.98 & 24.9742-C30 & 24.3689-C30 \\
\hline & 114.87-C7 & 114.799-C13 & 20.07 & 21.5326-C27 & 21.083-C27 \\
\hline & 113.289-C11 & 112.649-C11 & & 18.302-C35 & 17.7894-C35 \\
\hline \multirow[t]{2}{*}{53.47} & $50.3871-\mathrm{C} 21$ & 44.1719-C21 & & $12.926-\mathrm{C} 43$ & 13.0837-C43 \\
\hline & & & & 7.0408-C47 & 6.1516-C47 \\
\hline
\end{tabular}

$R^{2}=0.9985$ and $\mathrm{RMSD}=8.615417071 \mathrm{ppm}$ for Monomer 1 and $R^{2}=0.9981$ and $\mathrm{RMSD}=9.136034 \mathrm{ppm}$ for Monomer 2.

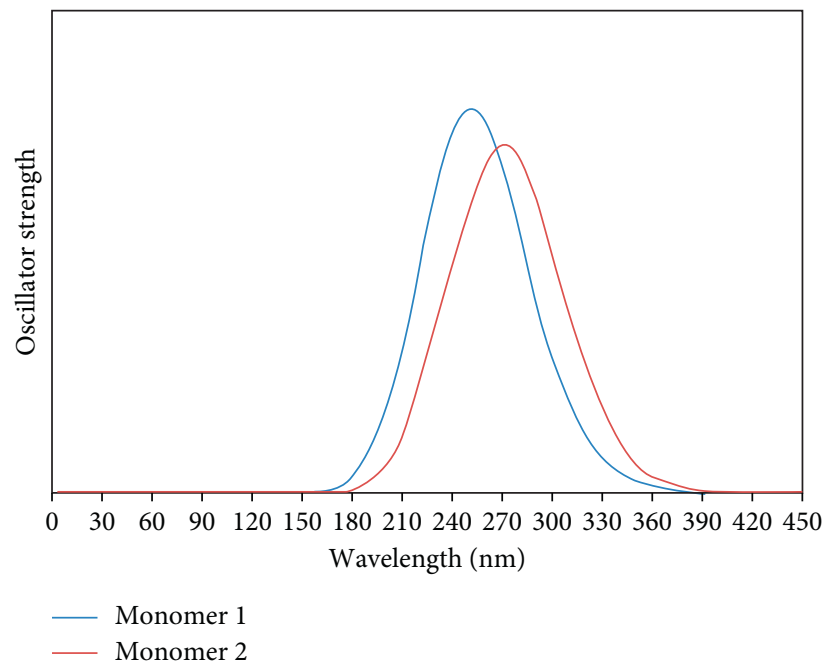

FIgURE 7: The simulated UV-Vis analysis of nateglinide in methanol, Monomer 1 and Monomer 2.

$212.63 \mathrm{~nm}$ with $5.8310 \mathrm{eV}$ value of excitation energy and 0.0133 value of oscillator strength for Monomer 2 . The other computed wavelengths are given as $228.64 \mathrm{~nm}, 219.70 \mathrm{~nm}$, $216.37 \mathrm{~nm}, 212.63 \mathrm{~nm}$, and $210.78 \mathrm{~nm}$.

3.5. HOMO-LUMO Analyses. The computed molecular energies for Monomer I and Monomer II were obtained as $E=-1020.83682668$ a.u. and $E=-1020.83864468$ a.u., respectively. The calculated dipole moments are 5.1216 and 2.3479 debye for Monomer I and Monomer II, respectively. By considering Monomer II, the structural, spectroscopic (IR, Raman, NMR, and UV-Vis), and HOMO-LUMO analyses for nateglinide were performed using theoretical computational methods. The relative energy between the two monomers is considerably low, and it has a value of $-0.99582 \mathrm{kcal} / \mathrm{mole}$. Owing to its more stable structure, dipole moment of monomer 2 is lower than monomer 1. The simulated HOMO and LUMO surfaces, energy values, and their shapes for the title molecule are given in Figure 8. The calculated HOMO and LUMO energy values were computed as $-6.9449 \mathrm{eV}$ and $-0.8923 \mathrm{eV}$ for Monomer 1 and $-6.8336 \mathrm{eV}$ and $-0.8101 \mathrm{eV}$ for Monomer 2 at the DFT/B3LYP/6-31G+(d, p) level, respectively.

\section{Conclusion}

The structural, spectroscopic (IR, Laser-Raman, NMR, and UV-Vis), and HOMO-LUMO analyses for nateglinide were performed using theoretical computational methods. The computed spectral properties were compared with the experimental data. After the conformational analysis, two molecular geometric forms at the lowest energies were optimized with the DFT/B3LYP/6-31G+(d, p) level. The results can be summarized as follows:

(i) The linear correlation coefficient $\left(R^{2}\right)$ value between the calculated and experimental [27] molecular geometric parameters was found as 0.975 for bond lengths $(\AA)$ and 0.9605 for bond angles $\left(^{\circ}\right)$, respectively, as given in Table 3.

(ii) As a result of the performed analyses, the linear correlation coefficient $\left(R^{2}\right)$ values between the experimental and computed vibrational frequencies of Monomer 1 and Monomer 2 for IR wavenumbers were found as $R^{2}=0.9981$ and $R^{2}=0.9980$, respectively.

(iii) The $R^{2}$ and RMSD values between the experimental and computed ${ }^{1} \mathrm{H}$ NMR chemical shifts were found 


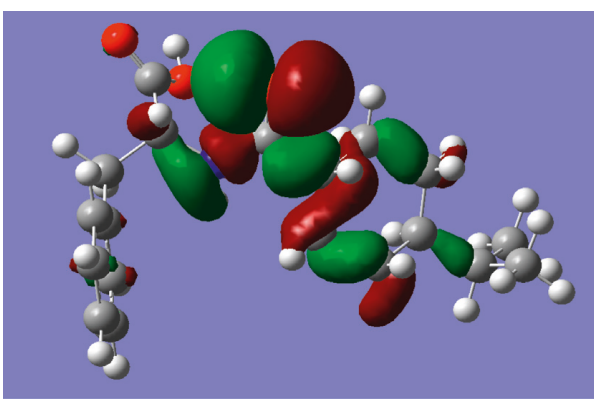

(a)

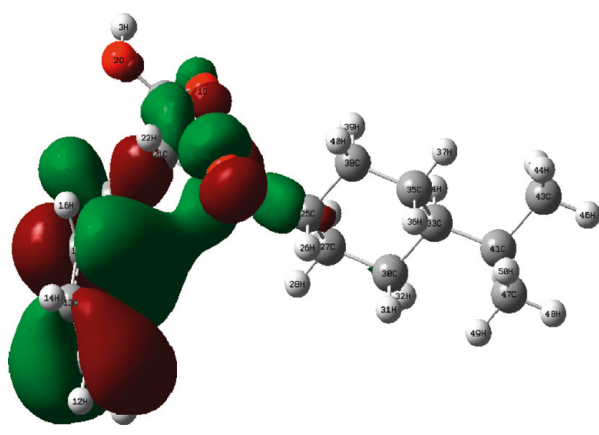

(c)

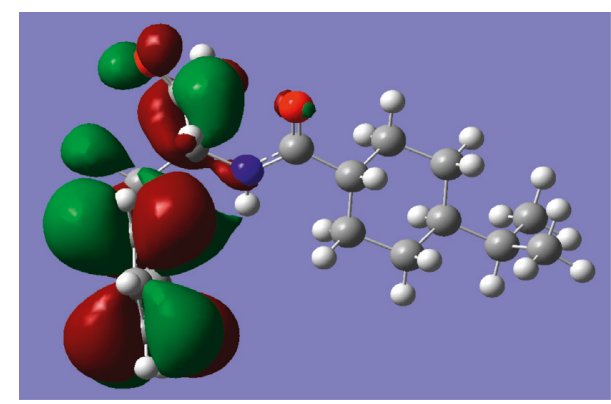

(b)

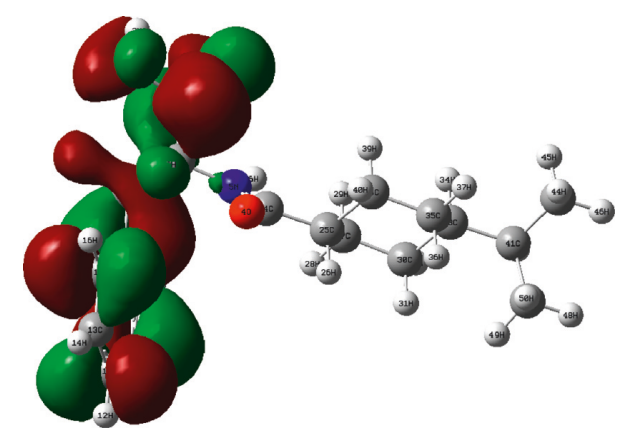

(d)

Figure 8: The HOMO and LUMO energy plots of nateglinide molecule. (a) Monomer 1: HOMO=-6.9449 eV. (b) Monomer 1: $\mathrm{LUMO}=-0.8923 \mathrm{eV}$. (c) Monomer 2: $\mathrm{HOMO}=-6.8336 \mathrm{eV}$. (d) Monomer 2: $\mathrm{LUMO}=-0.8101 \mathrm{eV}$.

as 0.9932 and $0.24 \mathrm{ppm}$ for Monomer 1 and as 0.9709 and 0.40 ppm for Monomer 2, respectively. The $R^{2}$ and RMSD values between the experimental and computed ${ }^{13} \mathrm{C}$ NMR chemical shifts were found as 0.9985 and $8.62 \mathrm{ppm}$ for Monomer 1 and as 0.9981 and 9.14 ppm for Monomer 2, respectively.

(iv) The major contributions for Monomer 1 were found as $\mathrm{H}-2->\mathrm{LUMO}$ (67\%), H-1->LUMO (12\%) H-3$>$ LUMO (6\%), H-2->L + 4 (5\%), and HOMO$>$ LUMO (2\%) for $216.85 \mathrm{~nm}$ wavelength. The major contributions for Monomer 2 were found as $\mathrm{H}-3$ $>$ LUMO (64\%), H-1->LUMO (10\%) H-1->L + 2 (6\%), HOMO->LUMO (3\%), and HOMO->L + 1 (4\%) for $212.63 \mathrm{~nm}$ wavelength.

\section{Data Availability}

The data used to support the findings of this study are available from the corresponding author upon request.

\section{Additional Points}

(i) The optimized molecular geometry and molecular parameters of nateglinide molecule were investigated. (ii) Molecular structure of nateglinide molecule was studied using DFT. (iii) The complete assignments were performed on the basis of the PED. (iv) The vibrational wavenumbers (FT-IR and Laser-Raman) and UV-Vis spectroscopy were studied using experimental and theoretical methods. (v) The experimental and computed proton and carbon-13 NMR chemical shifts were determined. (vi) All the results were compared with experimental (FT-IR, FT-Raman, UV-Vis, and NMR) spectra.

\section{Conflicts of Interest}

The author declares that there are no conflicts of interest.

\section{Acknowledgments}

This work was supported by Bartin University Research Fund Project under the Project no. 2017-FEN-A-005. The author thanks Cankiri Karatekin University Research Center for NMR, FT-IR, and UV-Vis analysis and Niğde Ömer Halisdemir University Center Research Laboratory for Laser-Raman analysis. The valuable help of Assoc. Prof. Dr. Halil Gökçe, Assoc. Prof. Dr. Halil Oturak, Asst. Prof. Adnan Sağlam, Asst. Prof. Firdevs Banu Özdemir, Pharmaceutist Ali Ünsal Keskiner, and Res. Asst. Mecit Öge is also acknowledged.

\section{References}

[1] Novartis, Starlix ${ }^{\circledR}$ (nateglinide) tablets, http://www.pharma. us.novartis.com/product/pi/pdf/Starlix.pdf/, 2013.

[2] N. Nordisk, "Prandin ${ }^{\circledR}$ (repaglinide) tablets," July 2018, http://www.novo-pi.com/prandin.pdf/.

[3] J. S. Grant and L. J. Graven, "Progressing from metformin to sulfonylureas or meglitinides," Workplace Health and Safety, vol. 64, no. 9, pp. 433-439, 2016.

[4] J. F. McLeod, "Clinical pharmacokinetics of nateglinide," Clinical Pharmacokinetics, vol. 43, no. 2, pp. 97-120, 2004.

[5] N. R. Pani, L. K. Nath, S. Acharya, and B. Bhuniya, "Application of DSC, IST, and FTIR study in the compatibility 
testing of nateglinide with different pharmaceutical excipients," Journal of Thermal Analysis and Calorimetry, vol. 108, no. 1, pp. 219-226, 2012.

[6] R. Landgraf, "Meglitinide analogues in the treatment of type 2 diabetes mellitus," Drugs \& Aging, vol. 17, no. 5, pp. 411-425, 2000.

[7] R. E. Pratley, J. E. Foley, and B. E. Dunning, "Rapid acting insulinotropic agents: restoration of early insulin secretion as a physiologic approach to improve glucose control," Current Pharmaceutical Design, vol. 7, no. 14, pp. 1375-1397, 2001.

[8] https://www.sigmaaldrich.com/catalog/product/usp/1457607? lang=en\&region=TR\&cm_sp=Insite-_-prodRecCold_xviews-_prodRecCold10-2, April 2018.

[9] S. Jain, A. Bhandari, and S. Purohit, "Spectrophotometric determination of nateglinide in bulk and tablet dosage forms," Asian Journal of Pharmaceutics, vol. 3, no. 3, 2009.

[10] G. R. Babu, A. L. Rao, S. L. Surekha, T. Kalapraveen, and P. S. Rao, "Spectrophotometric methods for estimation of Nateglinide in bulk drug and its dosage form," International Journal of Pharmaceutical, Chemical And Biological Sciences, vol. 3, no. 4, pp. 1160-1164, 2013.

[11] G. R. Babu, A. L. Rao, S. L. Surekha, T. Kalapraveen, and P. S. Rao, "Quantitative estimation of nateglinide in pharmaceutical dosage forms by visible spectrophotometry," IJRPC, vol. 3, no. 4, pp. 803-807, 2013.

[12] G. Bruni, V. Berbenni, C. Milanese et al., "Determination of the nateglinide polymorphic purity through dsc," Journal of Pharmaceutical and Biomedical Analysis, vol. 54, no. 5, pp. 1196-1199, 2011.

[13] R. Guardado-Mendoza, A. Prioletta, L. M. Jiménez-Ceja, A. Sosale, and F. Folli, "State of the art paper the role of nateglinide and repaglinide, derivatives of meglitinide, in the treatment of type 2 diabetes mellitus," Archives of Medical Science, vol. 5, pp. 936-943, 2013.

[14] A. P. Rani, C. B. Sekaran, N. Archana, P. S. Teja, and B. Aruna, "Vis-spectrophotometric methods for the determination of nateglinide," International Journal of Chemical Sciences, vol. 7, no. 3, pp. 1642-1652, 2009.

[15] P. Goyal, D. Rani, and R. Chadha, "Exploring structural aspects of nateglinide polymorphs using powder x-ray diffraction," International Journal of Pharmacy and Pharmaceutical Sciences, vol. 9, no. 10, pp. 119-127, 2017.

[16] M. Remko, "Theoretical study of molecular structure, pKa, lipophilicity, solubility, absorption, and polar surface area of some hypoglycemic agents," Journal of Molecular Structure: THEOCHEM, vol. 897, no. 1-3, pp. 73-82, 2009.

[17] M. Karakaya, Y. Sert, M. Kürekçi, B. Eskiyurt, and Ç. Çırak, "Theoretical and experimental investigations on vibrational and structural properties of tolazamide," Journal of Molecular Structure, vol. 1095, pp. 87-95, 2015.

[18] T. Özdemir and H. Gökce, "FT-IR, Raman, and NMR spectroscopy and DFT theory of Glimepiride molecule as a Sulfonylurea compound," Journal of Applied Spectroscopy, vol. 85, no. 3, pp. 560-572, 2018.

[19] C. M. Xavier, Analytical studies on some anti-diabetic drugs, Ph.D. thesis, University of Mysore, Mysore, Karnataka, India, 2015.

[20] A. D. Becke, "Density-functional thermochemistry. III. The role of exact exchange," Journal of Chemical Physics, vol. 98, no. 7, pp. 5648-5652, 1993.

[21] C. Lee, W. Yang, and R. G. Parr, "Development of the ColleSalvetti correlation-energy formula into a functional of the electron density," Physical Review B, vol. 37, no. 2, pp. 785789, 1988
[22] A. Frish, A. B. Nielsen, and A. J. Holder, Gauss View User Manual, Gaussian Inc., Pittsburg, PA, USA, 2001.

[23] M. J. Frisch, G. W. Trucks, H. B. Schlegel et al., Gaussian 09, Revision, A.1, Gaussian Inc., Wallingford, CT, USA, 2009.

[24] Gaussian Website, Visualizing Molecules \& Reactions with Gaussview 5, August 2016, http://www.gaussian.com/g_prod/ gv5.htm.

[25] M. H. Jamr'oz, "Vibrational energy distribution analysis VEDA4," Spectrochimica Acta Part A: Molecular and Biomolecular Spectroscopy, vol. 114, pp. 220-230, 2004.

[26] M. O’boyle, A. L. Tenderholt, and K. M. Langner, "Cclib: a library for package-independent computational chemistry algorithms," Journal of Computational Chemistry, vol. 29, no. 5, pp. 839-845, 2008.

[27] L. Tessler and I. Goldberg, "Bis(nateglinide) hydronium chloride, and its unique self-assembly into extended polymeric arrays via $\mathrm{O}-\mathrm{H} . . . \mathrm{O}, \mathrm{N}-\mathrm{H}$...Cl and $\mathrm{O}-\mathrm{H} . . . \mathrm{Cl}$ hydrogen bonds," Acta Crystallographica Section C Crystal Structure Communications, vol. 61, no. 12, pp. 738-740, 2005.

[28] V. Jain, D. K. Dhaked, Y. Kasetti, and P. V. Bharatam, "Computational study on the conformational preferences in nateglinide," Journal of Physical Organic Chemistry, vol. 25, no. 8, pp. 649-657, 2012.

[29] https://cccbdb.nist.gov/vibscalejust.asp, March 2018.

[30] N. Pandey, A. N. Sah, and K. Mahara, "Formulation and evaluation of floating microspheres of nateglinide," International Journal of Pharma Sciences aand Research, vol. 7, no. 11, pp. 453-464, 2016.

[31] M. Sa'id, A. S. Bayero, and U. L. Ali, "Determination of infrared, raman, $\left({ }^{1} \mathrm{H}\right.$ and $\left.{ }^{13} \mathrm{C}\right)-\mathrm{Nmr}$ spectra and density of state of nateglinide oral antidiabetic drug," International Journal of Nanomedicine and Nanosurgery, vol. 4, no. 1, 2018.

[32] A. Patil, B. G. Desai, H. N. Shivakumar, and Purvang, "enhancement of nateglinide solubility and dissolution rate," Research Journal of Pharmacy and Technology, vol. 4, no. 7, pp. 1159-1164, 2011.

[33] A. Rajasekaran, S. Murugesan, M. K. A. Hathi et al., "Spectrophotometric and chromatographic assay of Nateglinide," Indian Journal Pharmaceutical Sciences, vol. 66, p. 806, 2004. 

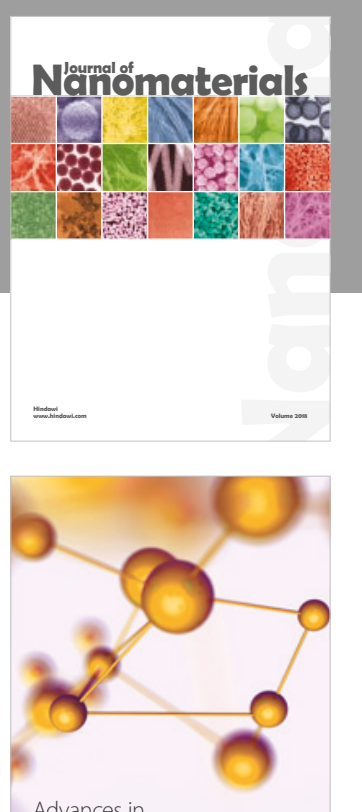

Physical Chemistry
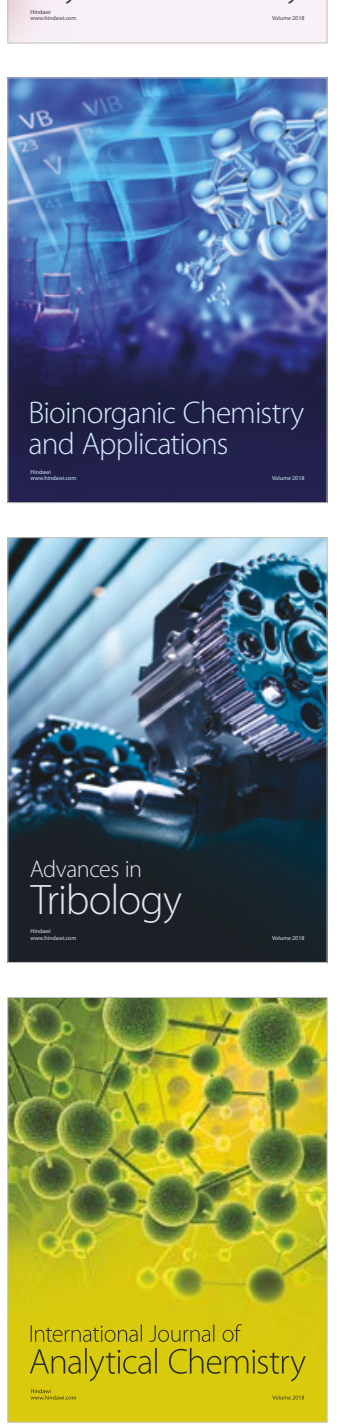

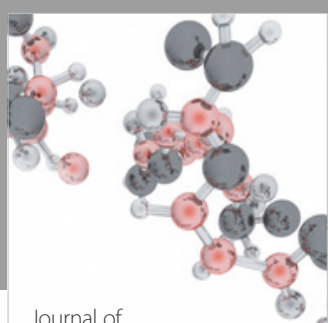

Analytical Methods

in Chemistry

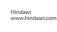

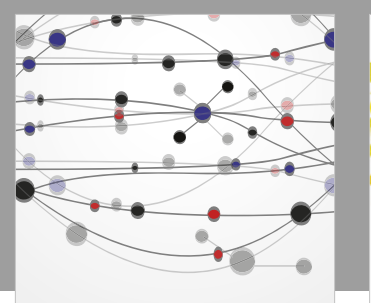

The Scientific World Journal

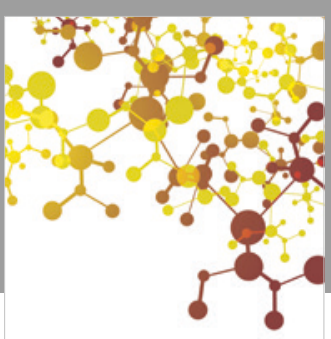

Journal of

Applied Chemistry
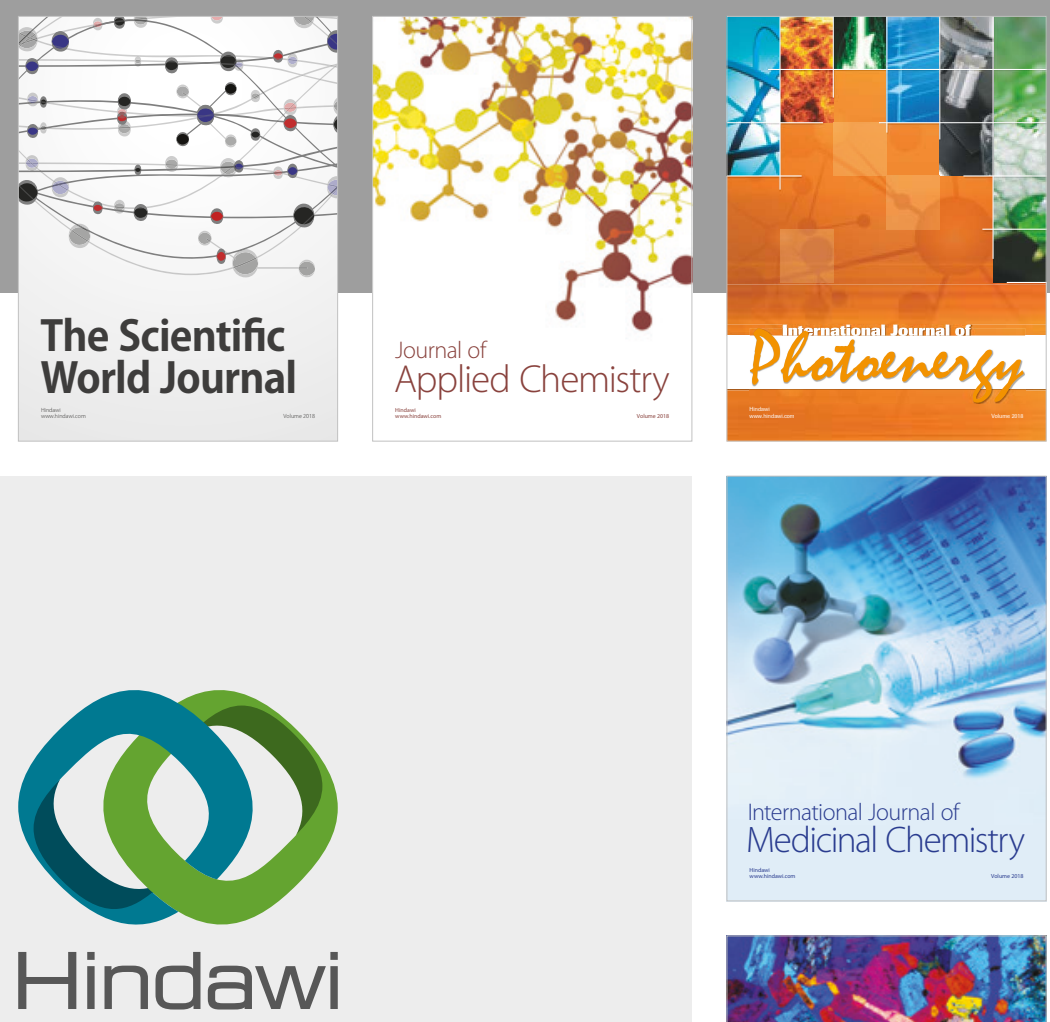

Submit your manuscripts at

www.hindawi.com
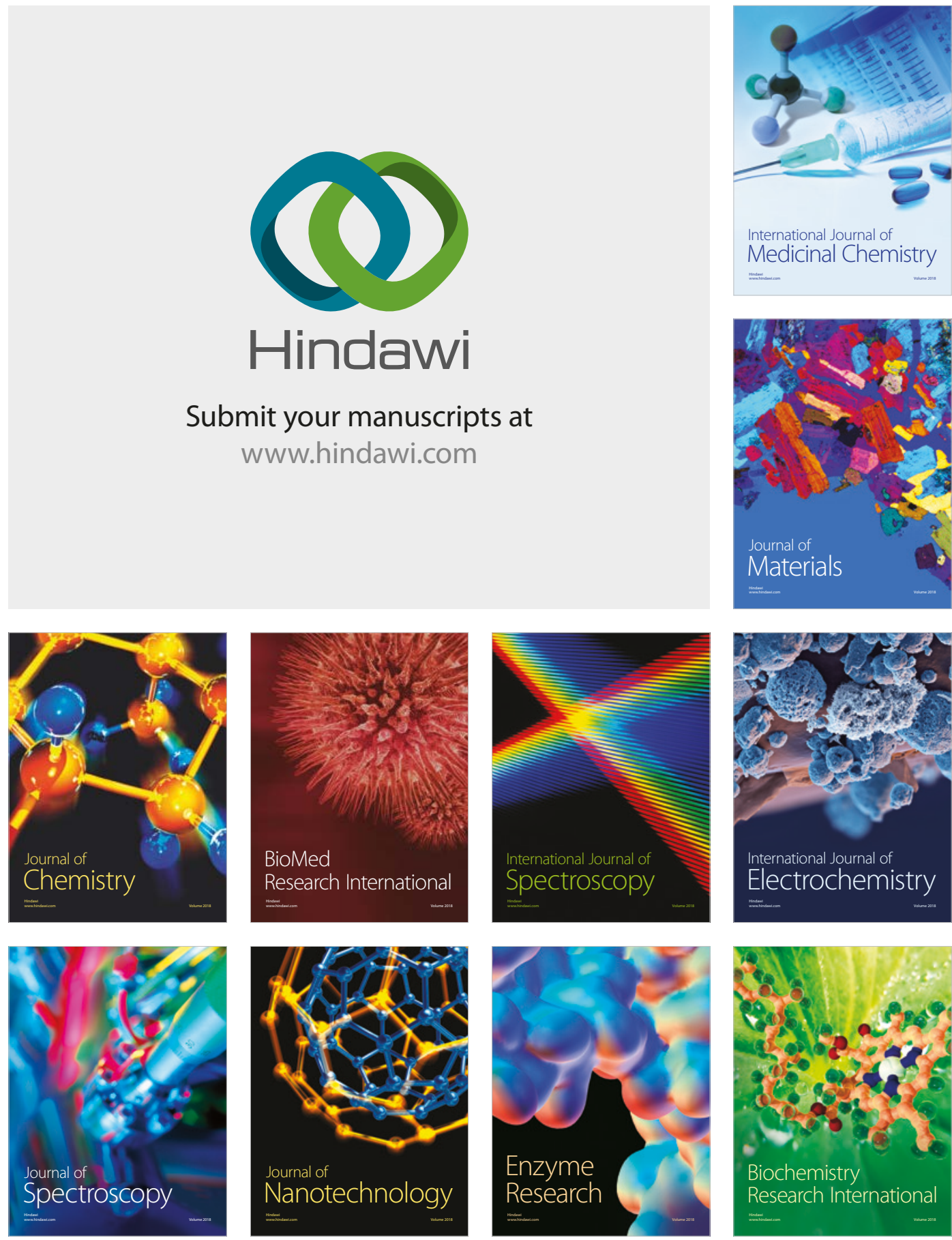
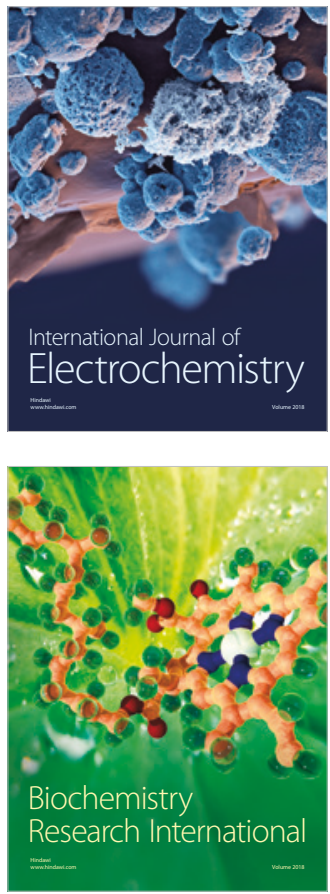